\title{
Blockwise empirical likelihood for spatial Markov model assessment
}

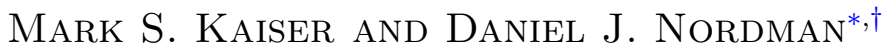

We propose a class of procedures for the assessment of Markov random field models based on spatial blockwise empirical likelihood (SBEL). These SBEL procedures have discernible asymptotic properties and provide a means for testing particular assumptions made in model formulation, such as distributional form and neighborhood structure. Based on the ideas of empirical likelihood, SBEL procedures are nonparametric in that they do not depend on parameterized likelihood functions per se. However, the moment conditions that are the focus of SBEL tests may themselves reflect data behaviors that are dictated by specific components of fully parametric models. Model assessment based on SBEL, then, produces completely data-driven procedures for testing the assumptions of parameterized Markov random field models. The procedures require two sets of moment conditions that are formulated as estimating functions. One set of estimating functions provides identification of parameter values and the other serves to define aspects of model behavior that we wish to assess relative to the behavior of observed data. We illustrate the use of SBEL procedures using null (assumed) models that have Gaussian and binary conditional distributions when data are simulated from both these, and other, models. Among other results, we demonstrate that an appropriate SBEL procedure is capable of detecting incongruence between assumed and true neighborhood structure on a regular lattice.

AMS 2000 SUBJECT CLASSifications: Primary 62M30, 62G09; secondary 62F03.

KeYwords AND PHRASES: Estimating functions, Markov random fields, Pseudo-likelihood.

\section{INTRODUCTION}

Empirical likelihood (EL) is a statistical methodology for formulating likelihood-type inference without specifying a joint parametric distribution for the data. For independent data, Owen [32, 33] first proposed the method for inference on mean parameters and certain $M$-estimators, and Qin and Lawless [37] extended EL in an important, broad direction by allowing estimating functions and general parameters

* Research partially supported by NSF grant no. DMS 0906588.

${ }^{\dagger}$ Corresponding author.

satisfying moment conditions. Recently, Nordman [29] developed an EL approach for inference with stationary, spatial lattice data for testing and estimation of a variety of spatial parameters, such as means, smooth model functions of means, as well as parameters specified by estimating functions. This approach, called spatial blockwise empirical likelihood (SBEL), represents a spatial extension of general EL methods for time series based on "data blocking" techniques, where blocks of data serve to capture the underlying (usually unknown) dependence structure by preserving groups of neighboring observations. For weakly dependent time processes, Kitamura [21] first proposed a data-block-based, or "blockwise," EL for weakly dependent time series in the framework of estimating functions, which has been successfully applied in a variety of time series inference scenarios, such as goodness-of-fit tests [10], several types of regression [4, 39, 40, 43], fitting autoregressive [8] and non-linear [5] models, quantile and density estimation $[9,25]$ and for various dependence structures such as negative/positive association [44] or long-memory [31]. Data-blocking concepts have also extended bootstrap and subsampling methodologies from independent to dependent data, such as the moving block bootstrap of Carlstein [7], Künsch [23] and Liu and Singh [26] for time series, and the spatial subsampling methods of Politis and Romano [35] and Sherman [42]; see $[24,36]$ for detailed overviews of block resampling or subsampling methods for temporal and spatial data.

In previous works, Nordman [29] and Nordman and Caragea [30] have investigated the estimation properties of SBEL for spatial parameters, such as means and variograms. The purpose of this manuscript differs in that we seek to demonstrate and investigate SBEL for spatial model assessment. In particular, we consider the specific, but challenging, problem of assessing whether or not a fitted spatial Markov random field model provides an adequate description of spatial data, which depends on factors such as the specified model class and neighborhood structure in the Markov model. In the SBEL framework, this issue can be addressed through formal tests of certain types of moment conditions. In econometrics, much research has focused on EL, and relatedly generalized method of moments estimators, for testing moment restrictions and conducting model diagnostics; see, for example, [5, 22, 28]. However, in contrast to the time series case, the potential of EL for testing moment conditions and performing formal data-structure diagnostics 
with spatial observations has not previously been explored. Established theory suggests that the SBEL method should be asymptotically valid for these purposes with stationary lattice data (cf. [29]), but the practical effectiveness of the method for using moment tests to assess models is a topic we wish to explore here, focusing on Markov random field models for concreteness.

We also note that tests for general spatial dependence structures, such as isotropy or covariance separability, have been developed in the literature, with some based on datablocking methods such as the block bootstrap or spatial subsampling (cf. [14, 27, 45]). In principle, the SBEL method could be used for similar tests and more, going beyond the particular assessments of Markov random field models considered here. This is because inference in the spatial EL is based on a likelihood function which has a common construction for any estimating functions used to identify spatial parameters or dependence conditions. The likelihood function can be maximized for point estimators, chi-square calibrated to set confidence regions, or applied to moment tests (also with a chi-square calibration). Hence, applying the SBEL method to different spatial problems requires only specifying appropriate estimating functions to prescribe the inference scenario. Additionally, the SBEL method for testing does not directly require any variance estimates in its implementation, which is useful in spatial settings where standard errors for test statistics can be difficult to obtain under potentially complicated spatial dependence structures.

The rest of the manuscript is organized as follows. In Section 2, we describe a large class of spatial Markov random field models, having conditionally specified distributions, and develop general estimating functions that can be used in the SBEL method to perform model assessment. In addition to estimating functions used for fitting the models, we consider two fundamental types of additional estimating functions for assessing conditional model specifications: one based on conditional moments and the other based on conditional independence. Section 3 provides the construction of the SBEL ratio as well as a distributional result for formally testing Markov model assumptions, entailing the SBEL ratio has a chi-squared limit when certain model conditions hold. Sections 4 and 5 evaluate and summarize the simulation performance of the SBEL method for assessing conditional Gaussian and conditional binary spatial Markov models, respectively. Section 6 provides a data illustration of the method for evaluating neighborhood selection in modeling a plant disease. In Section 7, we provide concluding remarks and discussion on the use of SBEL in model assessment.

\section{SBEL FOR ASSESSING SPATIAL MARKOV MODELS}

To understand the implementation of the spatial EL method, an important aspect is establishing a set of appropriate estimating functions with respect to the spatial data at hand. Because we wish to assess Markov random field models, which have a specification in terms of conditional distributions, the estimating functions should appropriately incorporate information on the form of such conditional distributions (and their corresponding parameters) and connect these to the data. We describe a general formulation of this in the following.

\subsection{A class of Markov random field models}

We assume data from a real-valued, strictly stationary process $\left\{X(\mathbf{s}): \mathbf{s} \in \mathbb{Z}^{2}\right\}$ on the planar integer grid $\mathbb{Z}^{2}$, and will later describe a more concrete sampling framework where observations are collected at sites within a spatial sampling region. But, for now, we are interested in considering a conditionally specified or Markov model for the spatial process $\left\{X(\mathbf{s}): \mathbf{s} \in \mathbb{Z}^{2}\right\}$. For clarity, we will use exclusively bold font for denoting spatial locations on the grid $\mathbb{Z}^{2}$ (i.e., $\mathbf{s} \in \mathbb{Z}^{2}$, zero vector $\mathbf{0}_{2} \in \mathbb{Z}^{2}$ ).

In such models, a conditional distribution for each random variable, $X(\mathbf{s})$, $\mathbf{s} \in \mathbb{Z}^{2}$, is typically expressed through a neighborhood structure. For instance, using some fixed template region $\mathcal{M} \subset \mathbb{Z}^{2} \backslash\left\{\mathbf{0}_{2}\right\}$ of finite size, many common neighborhood structures for $X(\mathbf{s})$ can be formulated as $\mathcal{N}(\mathbf{s})=\mathbf{s}+\mathcal{M}$. Neighborhoods which fit this form include so-called 4- or 8-nearest neighbor schemes, pictured below (where $*$ denotes a neighbor of $\mathbf{s} \in \mathbb{Z}^{2}$ ),
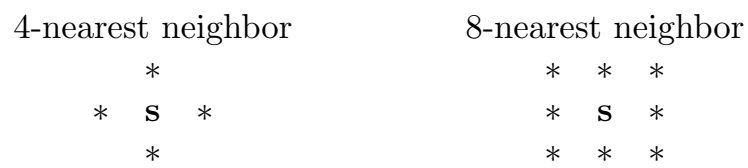

for which $\mathcal{M}=\left\{(-1,0)^{\prime},(1,0)^{\prime},(0,1)^{\prime},(0,-1)^{\prime}\right\}$ and $\mathcal{M}=$ $\left\{(u, v)^{\prime} \in \mathbb{Z}^{2}: \max \{|u|,|v|\}=1\right\}$, respectively. Throughout, we shall assume such neighborhood structures $\mathcal{N}(\mathbf{s})=\mathbf{s}+\mathcal{M}$ based on a template $\mathcal{M}$, which is reasonable for many spatial Markov models for data on regular lattices.

Under a Markov assumption, the conditional cumulative distribution of an observation $X(\mathbf{s}), \mathbf{s} \in \mathbb{Z}^{2}$ can be written as

$$
\begin{aligned}
& P\left(X(\mathbf{s}) \leq x \mid X(\mathbf{t}), \mathbf{t} \neq \mathbf{s} \in \mathbb{Z}^{2}\right) \\
& \quad=P(X(\mathbf{s}) \leq x \mid X(\mathbf{t}), \mathbf{t} \in \mathcal{N}(\mathbf{s})), \quad x \in \mathbb{R},
\end{aligned}
$$

which is functionally dependent only on neighboring observations (cf. [11], ch. 6). In the model development, we allow this conditional distribution for $X(\mathbf{s})$ to potentially depend on a $p$-dimensional parameter vector $\theta \in \Theta \subset \mathbb{R}^{p}$ and be expressed in terms of a conditional probability density function (in the continuous case) or conditional probability mass function (in the discrete case) written as

$$
f_{\theta}(x \mid\{X(\mathbf{t}): \mathbf{t} \in \mathcal{N}(\mathbf{s})\}), \quad x \in \mathbb{R},
$$

having a common form for all variables $X(\mathbf{s}), \mathbf{s} \in \mathbb{Z}^{2}$. Besag [2] introduced several conditional distributions of this 
form involving exponential family models, such as the autonormal model where the conditional density of $X(\mathbf{s}) \mid\{X(\mathbf{t})$ : $\mathbf{t} \in \mathcal{N}(\mathbf{s})\}$ in (2) is Gaussian with conditional variance $\tau^{2}>$ 0 and conditional mean $\mu(\mathbf{s})=\alpha+\eta \sum_{\mathbf{t} \in \mathcal{N}(\mathbf{s})}[X(\mathbf{t})-\alpha]$, the latter being a function of an unconditional mean parameter $E X(\mathbf{s})=\alpha \in \mathbb{R}$ and a dependence parameter $\eta$ (e.g., $|\eta|<1 / 4$ for a 4-nearest neighborhood structure); in this case, for example, $\theta=(\alpha, \eta, \tau)^{\prime}$. Arguably, the most common Markov model specifications involve some type of conditional Gaussian distributions [41], but other distributional forms are possible including beta [16, 19], binary [6], Poisson [2] and Winsorized Poisson [18]. Quite general conditional specifications are available for many exponential family models as well $[1,19]$.

\subsection{Estimating functions for model fit and assessment}

Similarly to the EL frameworks of Qin and Lawless [37] and Kitamura [21] for iid and dependent data, respectively, we require a set of estimating functions to link model forms to potential observations (under an expectation constraint). In particular, as the basis of spatial EL inference for conditionally specified distributions (2), we first require $p$-estimating functions to describe the involved model parameters $\theta \in \Theta \subset \mathbb{R}^{p}$. For this purpose, at a given location $\mathbf{s} \in \mathbb{Z}^{2}$, we use $p$ score-functions based on (2) to write estimating functions

$$
\begin{aligned}
& G_{p}\left[\mathcal{C}_{p}(\mathbf{s}) ; \theta\right] \\
& \quad \equiv \frac{\partial \log f_{\theta}(X(\mathbf{s}) \mid\{X(\mathbf{t}): \mathbf{t} \in \mathcal{N}(\mathbf{s})\})}{\partial \theta} \in \mathbb{R}^{p}
\end{aligned}
$$

evaluated at $\theta \in \Theta$ and a collection of variables $\mathcal{C}_{p}(\mathbf{s}) \equiv$ $\{X(\mathbf{t}): \mathbf{t}=\mathbf{s}$ or $\mathbf{t} \in \mathcal{N}(\mathbf{s})\}$ containing $X(\mathbf{s})$ and its neighbors under the model specification (2). For many Markov random fields based on exponential-family models, scorefunctions satisfy the moment condition

$$
E G_{p}\left[\mathcal{C}_{p}(\mathbf{s}) ; \theta_{0}\right]=0_{p} \in \mathbb{R}^{p}
$$

at the true parameter $\theta_{0}$ value. This moment restriction serves to identify the parameters in estimation and, in fact, these same score-based estimating functions are often used to produce maximum pseudo-likelihood estimators as the solution to $\sum_{i=1}^{n} G_{p}\left[\mathcal{C}_{p}\left(\mathbf{s}_{i}\right) ; \theta\right]=0_{p} \in \mathbb{R}^{p}$ (Besag, 1975).

The $p$ estimating functions in (3) suffice to produce point estimators and confidence regions for $\theta \in$ $\Theta \subset \mathbb{R}^{p}$ in the SBEL method. However, for purposes of model assessment, we need to augment (3) with an additional set of $q$-dimensional $(q>1)$ estimating functions $G_{a}\left[\mathcal{C}_{p}(\mathbf{s}), \mathcal{C}_{a}(\mathbf{s}) ; \theta\right] \in \mathbb{R}^{q}$, which can be functions of variables $\mathcal{C}_{p}(\mathbf{s})$ in addition to a potential collection of augmenting variables $\mathcal{C}_{a}(\mathbf{s})=\left\{X(\mathbf{t}): \mathbf{t} \in \mathcal{N}^{a u g}(\mathbf{s})\right\}$ defined by an augmentation neighborhood $\mathcal{N}^{\text {aug }}(\mathbf{s}) \subset \mathbb{Z}^{2}$ of location $\mathbf{s} \in \mathbb{Z}^{2}$. The augmentation neighborhood will typically depend on the question being assessed and may be empty as well, and we generally define $\mathcal{N}^{a u g}(\mathbf{s})=\mathbf{s}+\mathcal{A}$ as a translation of some finite template set $\mathcal{A} \subset \mathbb{Z}^{2}$ so that the augmentation neighborhoods have the same form for each location. We will describe examples of this in more detail below.

In general, then, letting $\mathcal{C}(\mathbf{s})=\mathcal{C}_{p}(\mathbf{s}) \cup \mathcal{C}_{a}(\mathbf{s})$, we suppose a total set of $q+p$ estimating functions $G[\mathcal{C}(\mathbf{s}) ; \theta] \equiv$ $\left(G_{p}\left[\mathcal{C}_{p}(\mathbf{s}) ; \theta\right], G_{a}\left[\mathcal{C}_{p}(\mathbf{s}), \mathcal{C}_{a}(\mathbf{s}) ; \theta\right]\right)^{\prime} \in \mathbb{R}^{q+p}$ which satisfy the moment condition

$$
E G\left[\mathcal{C}(\mathbf{s}) ; \theta_{0}\right]=0_{q+p}
$$

at the true parameter $\theta_{0}$. We mention two general and flexible strategies for formulating the additional estimating functions in $G_{a}[\cdot ; \theta]$.

Conditional moments From the conditional distribution of $X(\mathbf{s})$ in $(2)$ and integer $r \geq 1$, consider the $r$ th conditional moment, say $\mu_{r}(\mathbf{s} ; \theta) \in \mathbb{R}$, of this distribution, as a function of $\theta$ and the neighborhood variables $\{X(\mathbf{t}): \mathbf{t} \in$ $\mathcal{N}(\mathbf{s})\}$. One may then define $G_{a}\left[\mathcal{C}_{p}(\mathbf{s}), \mathcal{C}_{a}(\mathbf{s}) ; \theta\right]$ through a $q$-dimensional vector of differences $X^{r_{i}}(\mathbf{s})-\mu_{r_{i}}(\mathbf{s} ; \theta)$ based on moments $r_{1}, \ldots, r_{q}$, for which the moment condition (4) will hold under the (correctly specified) model (2). Alternatively, one could use centered moments, say $\mu_{r}^{c}(\mathbf{s} ; \theta) \in \mathbb{R}$, as well with estimating functions of form $\left[X(\mathbf{s})-\mu_{1}(\mathbf{s} ; \theta)\right]^{r}-$ $\mu_{r}^{c}(\mathbf{s} ; \theta)$. Note that, in using the conditional moments, we take $\mathcal{N}^{\text {aug }}(\mathbf{s})$ and $\mathcal{C}_{a}(\mathbf{s})=\emptyset$ as only the same variables $\mathcal{C}_{p}(\mathbf{s})$ from the score-functions (3) are needed. However, if any conditional moments already appear in the scorefunctions $G_{p}\left[\mathcal{C}_{p}(\mathbf{s}) ; \theta\right]$, these should not be repeated in defin$\operatorname{ing} G_{a}\left[\mathcal{C}_{p}(\mathbf{s}), \mathcal{C}_{a}(\mathbf{s}) ; \theta\right]$, because these add no new information about the conditional model.

Conditional covariances with non-neighbors Suppose a location $\mathbf{t} \neq \mathbf{s}$ is not an element of $\mathcal{N}(\mathbf{s})$ under the conditional specification (2); that is, $X(\mathbf{s})$ and $X(\mathbf{t})$ are not neighbors. Then, supposing $\mu_{1}(\mathbf{s} ; \theta) \in \mathbb{R}$ and $\mu_{1}(\mathbf{t} ; \theta) \in \mathbb{R}$ denote the first conditional moment of $X(\mathbf{s})$ and $X(\mathbf{t})$ under $(2)$, then by the Markov assumption and conditional independence,

$$
\begin{aligned}
E\left[X(\mathbf{s}) X(\mathbf{t})-\mu_{1}\left(\mathbf{s} ; \theta_{0}\right) \mu_{1}\left(\mathbf{t} ; \theta_{0}\right) \mid X(\mathbf{h}), \mathbf{h} \neq \mathbf{s}, \mathbf{t}\right] \\
=E[X(\mathbf{s}) X(\mathbf{t}) \mid X(\mathbf{h}), \mathbf{h} \in \mathcal{N}(\mathbf{s}) \cup \mathcal{N}(\mathbf{t})] \\
\quad-\mu_{1}\left(\mathbf{s} ; \theta_{0}\right) \mu_{1}\left(\mathbf{t} ; \theta_{0}\right)=0,
\end{aligned}
$$

implying that the marginal expectation of $X(\mathbf{s}) X(\mathbf{t})-$ $\mu_{1}(\mathbf{s} ; \theta) \mu_{1}(\mathbf{t} ; \theta)$ is also zero at the true parameter $\theta_{0}$. This suggests that, in designing $G_{a}[\cdot ; \theta]$, one could use estimating functions of the form $X(\mathbf{s}) X(\mathbf{t})-\mu_{1}(\mathbf{s} ; \theta) \mu_{1}(\mathbf{t} ; \theta)$ by choosing $\mathcal{N}^{\text {aug }}(\mathbf{s})$ to correspond to some subset of locations $\mathbf{t} \notin \mathcal{N}(\mathbf{s})$. The choice of augmenting locations, which are not neighbors of $\mathbf{s}$ under the model specification (2), would be driven by a particular model assessment issue. For example, suppose the neighborhoods $\mathcal{N}(\mathbf{s})$ have been chosen according to a 4-nearest neighbor strategy, and one would like to assess the adequacy of 
this against a possible 8-nearest neighbor strategy. One could select $\mathcal{C}_{a}(\mathbf{s})$ to contain the 8-nearest neighbors of $\mathbf{s}$ that are not also 4-nearest neighbors along with the 4-nearest neighbors of these as well, that is, $\mathcal{N}^{\text {aug }}(\mathbf{s})=$ $\left\{\mathbf{t}: \mathbf{t}=\mathbf{s}+(u, v)^{\prime}\right.$ or $\left.\mathbf{t} \in \mathcal{N}\left(\mathbf{s}+(u, v)^{\prime}\right),|u|=|v|=1\right\}$ which contains a total of 16 locations

$\begin{array}{ccccc} & \mathbf{h} & & \mathbf{h} & \\ \mathbf{h} & \mathbf{t} & \mathbf{n} & \mathbf{t} & \mathbf{h} \\ & \mathbf{n} & \mathbf{s} & \mathbf{n} & \\ \mathbf{h} & \mathbf{t} & \mathbf{n} & \mathbf{t} & \mathbf{h} \\ & \mathbf{h} & & \mathbf{h} & \end{array}$

as depicted above by locations $\mathbf{n}, \mathbf{t}$ and $\mathbf{h}$. An assessment estimating function for the question of 4-nearest versus 8nearest neighbors would then be

$$
\begin{aligned}
& G_{a}\left[\mathcal{C}_{p}(\mathbf{s}), \mathcal{C}_{a}(\mathbf{s}) ; \theta\right] \sum_{\substack{\mathbf{t}=\mathbf{s}+(u, v)^{\prime} \in \mathcal{N}^{\text {aug }}(\mathbf{s}),|u|=|v|=1}}\left[X(\mathbf{s}) X(\mathbf{t})-\mu_{1}(\mathbf{s} ; \theta) \mu_{1}(\mathbf{t} ; \theta)\right] \\
& \quad=
\end{aligned}
$$

having expectation zero (4) at $\theta_{0}$ under a correctly specified 4-nearest neighbor arrangement. Here, the locations denoted as "n" in the above picture are the 4-nearest neighbors of $\mathbf{s}$ and are needed to compute $\mu_{1}(\mathbf{s} ; \theta)$; the locations denoted by "t nearest neighbors and represent the locations summed over in (5); finally, the locations denoted as "h" above are additional locations needed to compute $\mu_{1}(\mathbf{t} ; \theta)$.

Alternatively, to simplify computation, one might define

$$
\mathcal{C}_{a}(\mathbf{s})=\left\{\mathbf{t}: \mathbf{t}=\mathbf{s}+(u, v)^{\prime},|u|=|v|=1\right\}
$$

and consider

$$
G_{a}\left[\mathcal{C}_{p}(\mathbf{s}), \mathcal{C}_{a}(\mathbf{s}) ; \theta\right]=\left[X(\mathbf{s})-\mu_{1}(\mathbf{s} ; \theta)\right] \sum_{\mathbf{t} \in \mathcal{N}^{a u g}(\mathbf{s})} X(\mathbf{t})
$$

which also has expectation zero under a correctly specified 4-nearest neighbor scheme but only involves variables at 9 locations in $\mathcal{C}(\mathbf{s})=\mathcal{C}_{p}(\mathbf{s}) \cup \mathcal{C}_{a}(\mathbf{s})$ rather than 17 (i.e., the "h" locations are no longer needed).

Because $q>1$, the estimating functions $G$ are said to "over-identify" $\theta \in \Theta \subset \mathbb{R}^{p}$ (cf. [37]) and this plays a role in the EL framework for testing the null hypothesis $H_{0}$ : " $E G\left[\mathcal{C}(\mathbf{s}) ; \theta_{0}\right]=0_{q+p}$ holds for some parameter $\theta_{0}$." Hence, formal tests with spatial EL are possible to assess whether or not spatial data support the conditional model properties described by the estimating functions under the moment condition (4). We next briefly recall the construction of the spatial blockwise EL (SBEL) function and a distributional result for conducting moment tests.

\section{SBEL CONSTRUCTION}

Let $D_{n} \subset \mathbb{R}^{2}$ denote a spatial sampling region and suppose the available data are $\left\{X(\mathbf{s}): \mathbf{s} \in D_{n} \cap \mathbb{Z}^{2}\right\}$ located at $\mathbb{Z}^{2}$ sites within $D_{n}$, where $\left|D_{n} \cap \mathbb{Z}^{2}\right|=n$ denotes the sample size of the observed process $\left\{X(\mathbf{s}): \mathbf{s} \in \mathbb{Z}^{2}\right\}$. Suppose further that, for each location $\mathbf{s} \in \mathbb{Z}^{2}$, there is a (finite) collection $\mathcal{C}(\mathbf{s})=\mathcal{C}_{p}(\mathbf{s}) \cup \mathcal{C}_{a}(\mathbf{s})$ of random variables such that estimating functions $G[\mathcal{C}(\mathbf{s}) ; \theta] \in \mathbb{R}^{q+p}$, based on parameters $\theta \in \Theta \subset \mathbb{R}^{p}$ in the conditional model specification (2), satisfy the moment condition (4). Correspondingly, we then have an observed spatial sample $\left\{\mathcal{C}(\mathbf{s}): \mathbf{s} \in \mathcal{S}_{n}\right\}$, where $\mathcal{S}_{n} \equiv\left\{\mathbf{s} \in \mathbb{Z}^{2}: \mathcal{N}(\mathbf{s}) \cup \mathcal{N}^{\text {aug }}(\mathbf{s}) \subset D_{n}\right\}$ denotes the collection of available sampling sites for the vector process $\left\{\mathcal{C}(\mathbf{s}): \mathbf{s} \in \mathbb{Z}^{2}\right\}$. Figure 1 provides an illustration of the difference between $\mathcal{S}_{N}$ and the original sampling sites $D_{n} \cap \mathbb{Z}^{2}$ for $X(\cdot)$.

To construct a SBEL function for $\theta$, we require spatial blocks of observed vectors $G[\mathcal{C}(\mathbf{s}) ; \theta], \mathbf{s} \in \mathcal{S}_{n}$, as follows. With a sequence $b \equiv b_{n}$ of positive integers, define general blocks of $b^{2}$ integers $\mathcal{B}_{b}(\mathbf{i}) \equiv\left\{\mathbf{s} \in \mathbb{Z}^{2}: \mathbf{s} \in \mathbf{i}+b \mathcal{U}\right\}, \mathbf{i} \in \mathbb{Z}^{2}$, by scaling the unit square $\mathcal{U}=(-1 / 2,1 / 2]^{2}$ by $b$; we shall comment more about conditions on the block scaling $b$ below, but blocks are intended to be smaller in size than $D_{n}$ or the sampling region for $\mathcal{C}(\cdot)$. Let $\left\{\mathbf{i}_{1}, \ldots, \mathbf{i}_{N}\right\} \equiv\left\{\mathbf{i} \in \mathbb{Z}^{2}: \mathcal{B}_{b}(\mathbf{i}) \subset\right.$ $\left.\mathcal{S}_{n}\right\}$ denote the index set of all integer-translated squares $\mathcal{B}_{b}(\cdot)$ lying completely inside the sampling sites $\mathcal{S}_{n}$ for the $\mathcal{C}(\cdot)$-observations. That is, $\left\{\mathcal{B}_{b}\left(\mathbf{i}_{1}\right), \ldots, \mathcal{B}_{b}\left(\mathbf{i}_{N}\right)\right\}$ represents a collection of $N$ (overlapping) blocks, each containing $b^{2}$ sampling sites of $\mathcal{C}(\cdot)$; see Figure $1(\mathrm{c})$ for an illustration. The data blocks serve to keep neighboring observations of $\mathcal{C}(\cdot)$ together in order to preserve the their underlying spatial dependence in the SBEL function to follow; the same idea applies in the time series setting of EL (cf. [21]).

Given a parameter value $\theta$, for each data block $j=$ $1, \ldots, N$, we compute the sample average of the estimating functions $\mathcal{G}\left(\mathbf{i}_{j} ; \theta\right)=b^{-2} \sum_{\mathbf{s} \in \mathcal{B}_{b}\left(\mathbf{i}_{j}\right)} G[\mathcal{C}(\mathbf{s}) ; \theta] \in \mathbb{R}^{q+p}$ over the $b^{2}$ observations of $\mathcal{C}(\cdot)$ within the block. Then, to assess the plausibility or likelihood of a given parameter value $\theta$ with respect to the estimating functions under the moment constraint (4), the SBEL function $L_{n}(\theta)$ and ratio $R_{n}(\theta)$ for $\theta \in \Theta$ are determined by

$L_{n}(\theta)$

$$
\begin{gathered}
\sup \left\{\prod_{j=1}^{N} p_{j}: p_{j} \geq 0, \sum_{j=1}^{N} p_{j}=1, \sum_{j=1}^{N} p_{j} \mathcal{G}\left(\mathbf{i}_{j} ; \theta\right)=0_{q+p}\right\}, \\
R_{n}(\theta)=\frac{L_{n}(\theta)}{(1 / N)^{N}} .
\end{gathered}
$$

The SBEL function for $\theta \in \Theta$ maximizes a multinomial likelihood based on probabilities $p_{j}$ assigned to each block average $\mathcal{G}\left(\mathbf{i}_{j} ; \theta\right)$ of estimating functions, under an expectation-type linear constraint that mimics the moment 
(a)

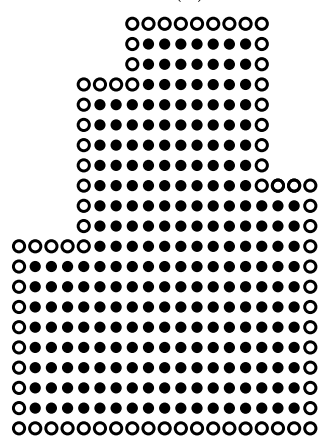

(b)

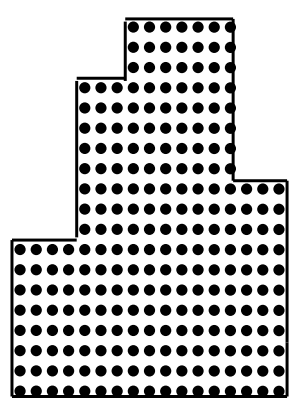

(c)

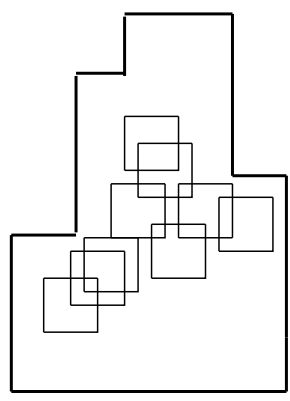

Figure 1. (a) Sampling region $D_{n}$ for original process $X(\mathbf{s})$ with sampling sites denoted as $\bullet$ or $\circ$. (b) Sampling sites $\mathcal{S}_{n}$ for observations $\mathcal{C}(\mathbf{s})=\left\{X(\mathbf{t}): \mathbf{t} \in \mathcal{N}(\mathbf{s}) \cup \mathcal{N}^{\text {aug }}(\mathbf{s})=\mathbf{s}+\left(\left\{\mathbf{0}_{2}\right\} \cup \mathcal{M} \cup \mathcal{A}\right)\right\}$, where $\mathcal{M}$ or $\mathcal{A}$ could be $\left\{(0,0)^{\prime},(-1,0)^{\prime},(1,0)^{\prime},(0,1)^{\prime},(0,-1)^{\prime}\right\}$ or $\left\{(u, v)^{\prime} \in \mathbb{R}^{2}:|u|,|v| \leq 1\right\}$; these sites match positions $\bullet$ in (a). (c) Illustration of data blocks within $\mathcal{S}_{n}$ as integer translates of scaled squares $b(-1 / 2,1 / 2]^{2}$.

condition (4). Without the expectation constraint in $L_{n}(\theta)$, the product has a maximum at the empirical distribution of the block averages (i.e., each $p_{j}=1 / N$ ), leading to the SBEL ratio in (7). The computation of the SBEL function for $\theta$ is the same as that for EL with iid data; see [33, 34, 37] for these computational details. In brief, when $0_{q+p}$ lies in the interior convex hull of $\left\{\mathcal{G}\left(\mathbf{i}_{j} ; \theta\right): j=1, \ldots, N\right\}$, then $L_{n}(\theta)$ is a positive, uniquely achieved maximum and (7) may be written as

(8) $L_{n}(\theta)=\prod_{j=1}^{N} p_{j}(\theta), \quad p_{j}(\theta)=\frac{1}{N\left[1+\lambda_{\theta}^{\prime} \mathcal{G}\left(\mathbf{i}_{j} ; \theta\right)\right]} \in(0,1)$

where $\lambda_{\theta} \in \mathbb{R}^{q+p}$ solves $\sum_{j=1}^{N} \mathcal{G}\left(\mathbf{i}_{j} ; \theta\right) /\left[1+\lambda^{\prime} \mathcal{G}\left(\mathbf{i}_{j} ; \theta\right)\right]=0_{q+p}$. Similarly to parametric likelihood, the SBEL function (7) can then be maximized to provide a point estimator $\hat{\theta}_{n}$ (a maximum SBEL estimator) for $\theta \in \Theta \subset \mathbb{R}^{p}$.

The next result gives an asymptotic chi-square $\chi_{q}^{2}$ distribution for the $\log$ SBEL ratio $R_{n}\left(\hat{\theta}_{n}\right)$ (evaluated at the maximum SBEL estimator) for testing the moment condition (4) as our basis of assessing conditional model specifications. The degrees of freedom $q$ are equal to the number of estimating functions in $G_{a}[\cdot ; \theta]$. The large-sample results are established in a so-called "increasing domain asymptotic" framework (cf. [11]), in which more gridded spatial observations $X(\cdot)$ are obtained by considering increasingly larger spatial sampling regions. The following result recasts Theorem 3 of Nordman [29] for the Markov model assessment problem here. We provide a rigorous statement that simplifies the underlying technical assumptions; these are generally mild for the model assessment problem, as will be briefly explained.

Theorem 3.1. Suppose an increasing domain framework with $b^{-1}+b^{2} / \sqrt{n} \rightarrow 0$ as $n \rightarrow \infty$ and that appropriate weak dependence conditions are satisfied for the stationary process $\left\{X(\mathbf{s}): \mathbf{s} \in \mathbb{Z}^{2}\right\}$ under the conditional distributions
(2). In addition, suppose that $G[\cdot ; \theta]$ is a smooth function of $\theta$ in a neighborhood of $\theta_{0}$, that $E\left\{\partial G[\mathcal{C}(\mathbf{s}) ; \theta] /\left.\partial \theta\right|_{\theta=\theta_{0}}\right\}$ has full column rank $p$ and the $(q+p) \times(q+p)$ matrix $\sum_{\mathbf{s} \in \mathbb{Z}^{2}} \operatorname{Cov}\left(G\left[\mathcal{C}\left(\mathbf{0}_{2}\right) ; \theta_{0}\right], G\left[\mathcal{C}(\mathbf{s}) ; \theta_{0}\right]\right)$ is positive definite.

Then, under the null hypothesis $H_{0}$ : "the moment condition (4) holds for some parameter $\theta_{0}$ under the conditional model specification (2)", the log-ratio satisfies

$$
-\frac{2}{b^{2}} \log R_{n}\left(\hat{\theta}_{n}\right) \stackrel{d}{\rightarrow} \chi_{q}^{2} \quad \text { as } n \rightarrow \infty .
$$

Note above that $b^{-2}$ represents an adjustment factor to the usual "-2 log-likelihood-ratio" due to the overlapping blocks of size $b^{2}$; a similar block adjustment exists for EL with time series (cf. Kitamura [21]). Regarding the block condition in the SBEL method, we require $b \rightarrow \infty$ as $n \rightarrow$ $\infty$ (so that blocks grow with increasing samples) but with $b^{2} / \sqrt{n} \rightarrow 0$, which entails that the size of the blocks is small relative to the overall sample size $n$. Often a block size of the form $b=C n^{1 / 5}$ for $C \in[1,2]$ can be appropriate for a range of dependence structures (e.g. [29] for interval estimation). We consider choice of block size in the simulations to follow.

In terms of the assumptions involved, the asymptotic limit generally requires the underlying process $\{X(\mathbf{s}): \mathbf{s} \in$ $\left.\mathbb{Z}^{2}\right\}$ to exhibit weak dependence, stipulated in terms of $\alpha$ mixing conditions (e.g. [12]). However, in the model assessment case here, if the conditional model specification (2) is such that the spatial process satisfies Dobrushin's uniqueness condition (e.g. [15]), then $\left\{X(\mathbf{s}): \mathbf{s} \in \mathbb{Z}^{2}\right\}$ is strongly mixing at an exponential rate and, hence, the $\alpha$-mixing conditions hold trivially. The smoothness conditions on the estimating functions are that, in a neighborhood of $\theta_{0}$, the partial derivatives $\partial G[\cdot, \theta] / \partial \theta, \partial^{2} G[\cdot, \theta] / \partial \theta \partial \theta^{\prime}$ are continuous in $\theta$ with cubed matrix norms that are bounded by integrable function; this holds for many exponential-family conditional distributions (2). Finally, in the increasing domain sampling framework, we suppose the sampling region $D_{n}=\lambda_{n} D_{0} \subset \mathbb{R}^{2}$ is obtained by inflating a template set 
$D_{0} \subset(-1 / 2,1 / 2]^{2}$ (containing an open neighborhood of $\mathbf{0}_{2}$ ) by an increasing positive sequence $\lambda_{n}$ of scaling factors. To avoid pathological regions, the number of integers (sampling sites) $n=\left|D_{n} \cap \mathbb{Z}^{2}\right|$ and the volume $\operatorname{vol}\left(D_{n}\right)$ are assumed to be asymptotically equivalent $n / \operatorname{vol}\left(D_{n}\right) \rightarrow 1$. Similar sampling structures have been considered by several authors [24, 42] in spatial resampling, which allows a variety of shapes for $D_{n}$. See [29] for more details.

\section{ASSESSMENT OF CONDITIONAL GAUSSIAN MODELS}

In this section, we summarize simulation studies performed to evaluate SBEL methods for model assessment. Here the null model (i.e., the model chosen for data fitting and subsequent assessment) is taken to be conditional Gaussian with a 4-nearest neighbor structure $\mathcal{N}(\mathbf{s})=\mathbf{s}+\mathcal{M}$, $\mathbf{s} \in \mathbb{Z}^{2}$ with $\mathcal{M}=\left\{(-1,0)^{\prime},(1,0)^{\prime},(0,1)^{\prime},(0,-1)^{\prime}\right\}$ as in (1). That is, in (2), the conditional distributional $X(\mathbf{s}) \mid\{X(\mathbf{t})$ : $\mathbf{t} \in \mathcal{N}(\mathbf{s})\}$ is normal with conditional variance $\tau^{2}>0$ and conditional mean

$$
\mu(\mathbf{s} ; \alpha, \eta)=\alpha+\eta \sum_{\mathbf{t} \in \mathcal{N}(\mathbf{s})}[X(\mathbf{t})-\alpha]
$$

involving (marginal mean) $\alpha \in \mathbb{R}$ and dependence parameter $|\eta|<0.25$. In this case, the score-based estimating functions (3) are equivalent to $p=3$ and

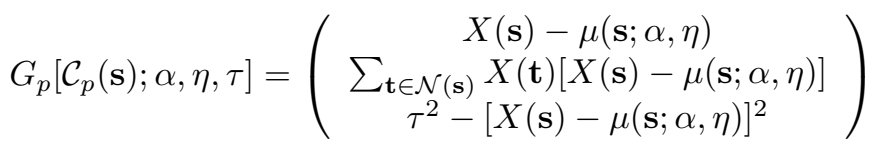

where again $\mathcal{C}_{p}(\mathbf{s})=\{X(\mathbf{t}): \mathbf{t}=\mathbf{s}$ or $\mathbf{t} \in \mathcal{N}(\mathbf{s})\}$. We consider over-identifying estimating functions $G_{a}[\cdot ; \alpha, \eta, \tau]$ along the two strategies outlined in Section 2.2. One set of functions we base on two conditional (centered) normal moments

$$
G_{a}\left[\mathcal{C}_{p}(\mathbf{s}), \mathcal{C}_{a}(\mathbf{s}) ; \alpha, \eta, \tau\right]=\left(\begin{array}{c}
{[X(\mathbf{s})-\mu(\mathbf{s} ; \alpha, \eta)]^{3}} \\
{[X(\mathbf{s})-\mu(\mathbf{s} ; \alpha, \eta)]^{4}-3 \tau^{4}}
\end{array}\right),
$$

taking $\mathcal{C}_{a}(\mathbf{s})=\emptyset$. Note that we use the third and fourth centered conditional moments, as the first and second such moments already appear in the score functions $G_{p}\left[\mathcal{C}_{p}(\mathbf{s}) ; \alpha, \eta, \tau\right]$ above. Under the null model, the moment condition (4) holds, and we refer to the resulting ("moment-based") SBEL test statistic as "SBEL.M" which will have a large-sample $\chi_{q=2}^{2}$ distribution under Theorem 3.1. We base another type of over-identifying estimating function on conditional covariances

$$
G_{a}\left[\mathcal{C}_{p}(\mathbf{s}), \mathcal{C}_{a}(\mathbf{s}) ; \alpha, \eta, \tau\right]=\sum_{\mathbf{t} \in \mathcal{N}^{a u g}(\mathbf{s})} X(\mathbf{t})[X(\mathbf{s})-\mu(\mathbf{s} ; \alpha, \eta)]
$$

Table 1. Spatial data-generating models considered in fitting the null (4-nearest neighbor) Gaussian model. As listed below, the conditional models have distributional forms, neighborhood structures, and parameters $\alpha=0, \tau=1$ and $\eta$

(determining weak or strong dependence), with the parameters having the same functional relationship as in the null Gaussian model

\begin{tabular}{cccc}
\hline \hline Distribution & Neighbors & Weak $\eta$ & Strong $\eta$ \\
\hline Conditional Gaussian & 4-nearest & 0.18 & 0.24 \\
Conditional Gaussian & 8-nearest & 0.09 & 0.12 \\
log-Conditional Gaussian & 4-nearest & 0.18 & 0.24 \\
log-Conditional Gaussian & 8-nearest & 0.09 & 0.12 \\
\hline
\end{tabular}

with $\mathcal{N}^{\text {aug }}(\mathbf{s})=\left\{\mathbf{s}+(u, v)^{\prime}:|u|=|v|=1\right\}$ as in (6) involving a sum over all variables in an 8-nearest neighbor structure around $\mathbf{s}$, that do not also appear in the 4nearest neighbor structure. We refer to the resulting ("conditional covariance-based") SBEL test statistic as "SBEL.C" which will have a large-sample $\chi_{q=1}^{2}$ distribution under Theorem 3.1. With the same augmenting variables $\mathcal{C}_{a}(\mathbf{s})$, we also considered another version, referred to as "SBEL.C2," that splits the above sum into two parts

$$
\begin{aligned}
G_{a} & \left.\mathcal{C}_{p}(\mathbf{s}), \mathcal{C}_{a}(\mathbf{s}) ; \alpha, \eta, \tau\right] \\
& =\left(\begin{array}{c}
\sum_{\mathbf{t}=\mathbf{s}+(u, v)^{\prime} \in \mathbb{Z}^{2},-u=v=1} X(\mathbf{t})[X(\mathbf{s})-\mu(\mathbf{s} ; \alpha, \eta)] \\
\sum_{\mathbf{t}=\mathbf{s}+(u, v)^{\prime} \in \mathbb{Z}^{2},-u=v=-1} X(\mathbf{t})[X(\mathbf{s})-\mu(\mathbf{s} ; \alpha, \eta)]
\end{array}\right) ;
\end{aligned}
$$

the resulting SBEL.C2 test statistic has $q=2$ degrees of freedom with performance results here that were very similar to SBEL.C. We shall illustrate this point, but focus on comparing SBEL.C and SBEL.M tests in the following.

To evaluate these test statistics in assessing the null model, we considered four spatial data-generating models with conditional specifications, each with two parameter settings entailing weaker and stronger forms of dependence. These models are listed in Table 1 . For each model, we generated data on two sampling regions, $30 \times 30$ and $50 \times 50$ (using 2,000 simulations in every case), and computed the SBEL test statistics using a block scaling of $b=1.5 n^{1 / 5}$ or $b=3 n^{1 / 5}$ where $n$ denotes the sample size (e.g., 900 or $2,500)$. The data-generations were based on a Gibbs sampler where, after a burn-in of 10,000 iterations, every 500th iteration was retained as a spatial sample.

Table 2 shows that the actual sizes of different SBEL tests, with block scaling $b=1.5 n^{1 / 5}$, matched nominal levels quite well under both weaker and stronger forms of dependence. This choice of block scaling also supports the simulation results in Nordman (2008), who found that block scaling $C n^{1 / 5}, C \in[1,2]$ tended to be a good range for SBEL confidence intervals in terms of coverage accuracy for mean parameters with other types of (non-conditionally specified) spatial processes. Figure 2 shows power curves to compare 
Gaussian, $30 \times 30$, SBEL.C

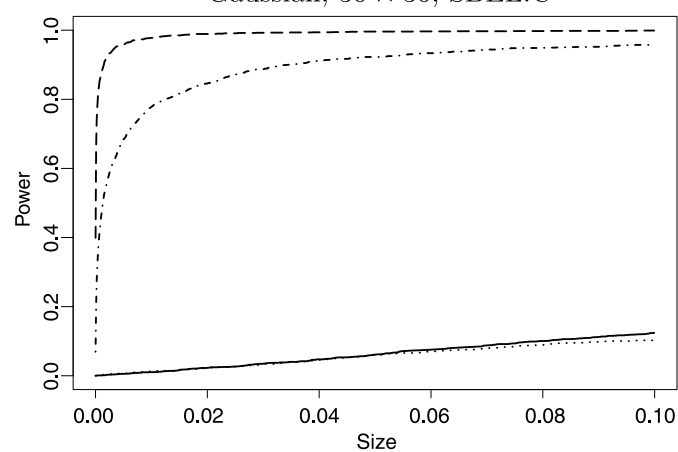

Gaussian, $50 \times 50$, SBEL.C

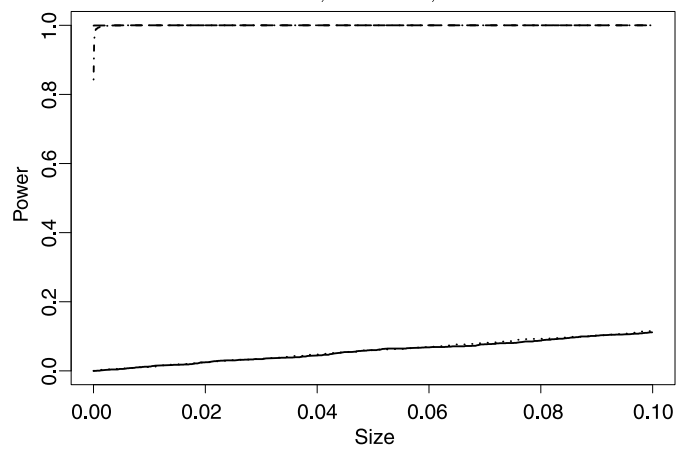

log-Gaussian, $30 \times 30$, SBEL.C

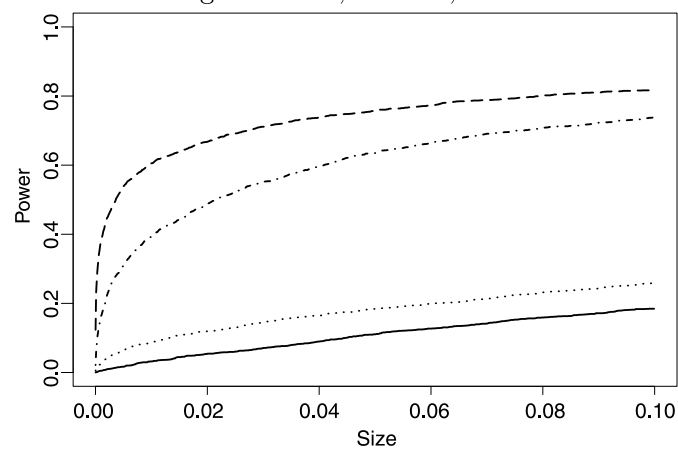

log-Gaussian, $50 \times 50$, SBEL.C

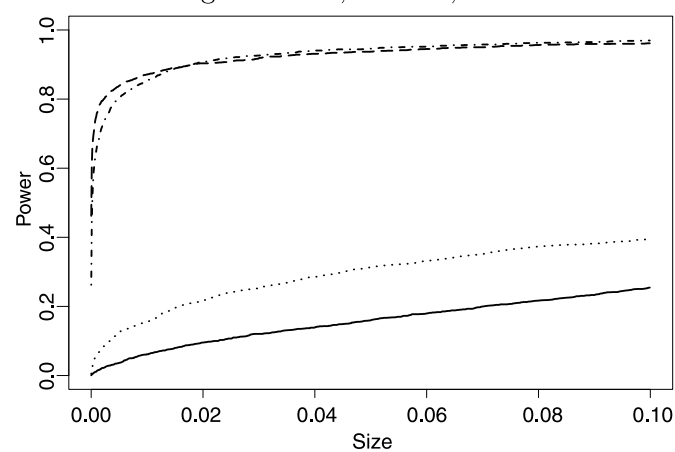

Gaussian, $30 \times 30$, SBEL.M

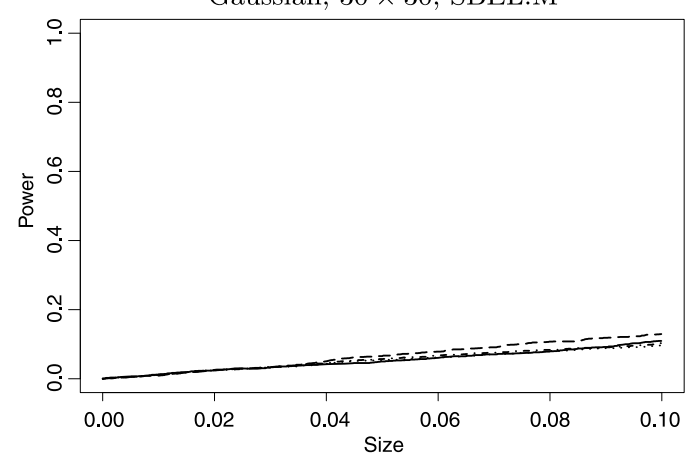

Gaussian, $50 \times 50$, SBEL.M

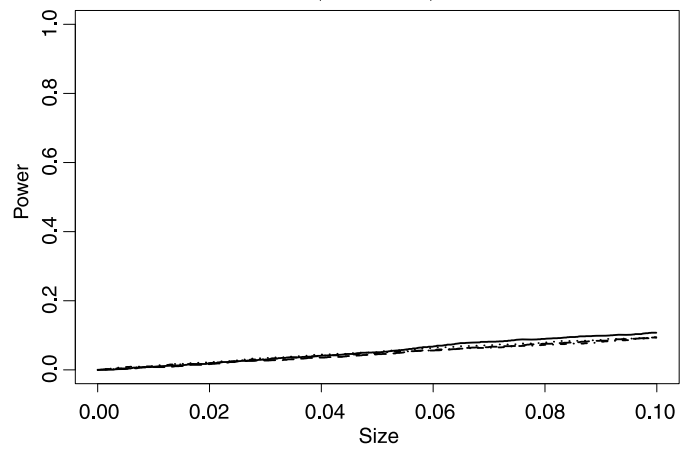

log-Gaussian, $30 \times 30$, SBEL.M

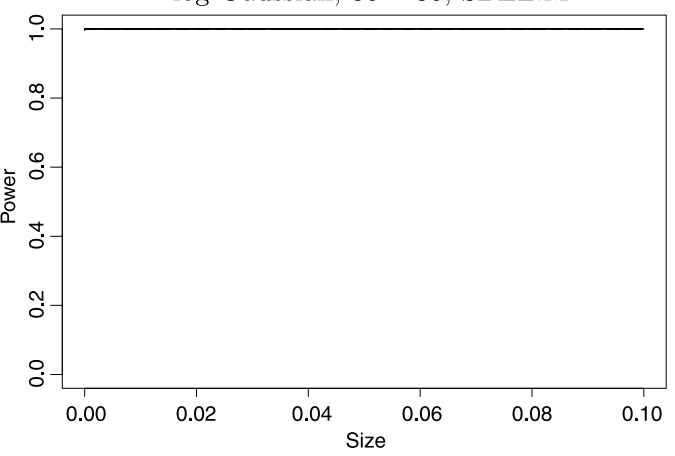

log-Gaussian, $50 \times 50$, SBEL.M

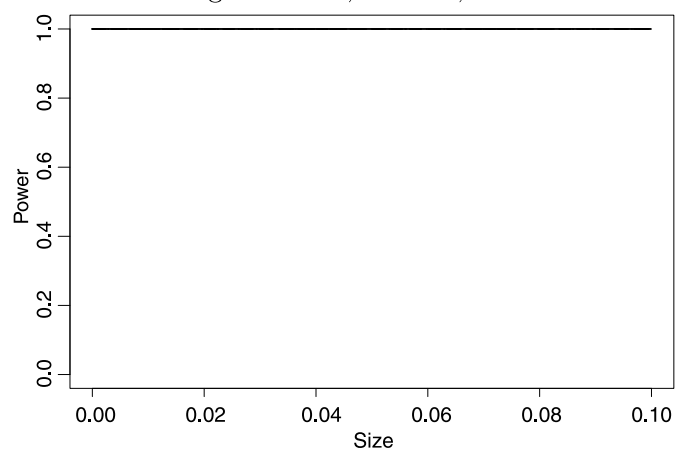

Figure 2. Power curves for testing the null (4-nearest neighbor conditional Gaussian) model. Plots correspond to conditionally specified data-generating models, SBEL tests $\left(b=1.5 n^{1 / 5}\right)$, and sampling regions; within a plot, curves represent either 4-nearest neighbor structure with weak (-) or strong ( ...) dependence or 8-nearest neighbor with weak (---) or strong (--) dependence in the data-generating model. 
Table 2. Actual sizes (expressed as percentages) of SBEL.M, SBEL.C, SBEL.C2 tests, at nominal levels of $10 \%, 5 \%$ and $1 \%$, for assessing the null (4-nearest neighbor conditional Gaussian) model when the data-generating model belongs to this model class with $\alpha=0, \tau=1$ and either weak $\eta=0.18$ or strong $\eta=0.24$ dependence parameters. The SBEL tests involve the block scaling $b=1.5 n^{1 / 5}$ here

\begin{tabular}{c|ccc|cccc|ccc|cc}
\hline \hline & \multicolumn{4}{c}{$30 \times 30$ region } & \multicolumn{3}{c}{ strong $\eta=0.24$} & \multicolumn{3}{c}{ weak $\eta=0.18$} & \multicolumn{1}{c}{$50 \times 50$ region } \\
Nominal & 10 & 5 & 1 & 10 & 5 & 1 & 10 & 5 & 1 & 10 & 5 & 1 \\
\hline SBEL.M & 10.1 & 5.0 & 1.3 & 9.7 & 5.4 & 1.1 & 10.8 & 5.1 & 0.9 & 9.5 & 5.2 & 1.2 \\
SBEL.C & 12.4 & 6.1 & 1.1 & 10.3 & 6.0 & 1.3 & 11.2 & 6.0 & 1.3 & 11.6 & 6.0 & 1.1 \\
SBEL.C2 & 11.0 & 5.0 & 1.0 & 10.9 & 5.3 & 1.1 & 11.1 & 5.6 & 0.9 & 11.6 & 5.6 & 0.9 \\
\hline
\end{tabular}

Table 3. Median parameter estimates for $(\alpha, \eta, \tau)$ in fitting the null (4-nearest neighbor conditional Gaussian) model with regular maximum pseudo-likelihood $(P)$ and maximum SBEL estimates from SBEL.M (M) and SBEL.C (C) functions (7) with

$b=1.5 n^{1 / 5}$; these are divided by different data-generating models involving weak and strong dependence and different sampling region sizes. In the case where data-generation occurs under the null model, median absolute deviations from the true parameters $(\alpha=0, \tau=1, \eta=0.18$ (weak) or 0.24 (strong)) appear in italics

\begin{tabular}{|c|c|c|c|c|c|c|c|c|c|c|c|c|}
\hline \multicolumn{13}{|c|}{ "Gaussian, 4-nearest neighbor structure } \\
\hline & \multicolumn{6}{|c|}{$30 \times 30$ region } & \multicolumn{6}{|c|}{$50 \times 50$ region } \\
\hline & \multicolumn{3}{|c|}{ weak dependence } & \multicolumn{3}{|c|}{ strong dependence } & \multicolumn{3}{|c|}{ weak dependence } & \multicolumn{3}{|c|}{ strong dependence } \\
\hline $\mathrm{P}$ & $\begin{array}{c}\alpha \\
-0.003 \\
0.053\end{array}$ & $\begin{array}{c}\eta \\
0.180 \\
0.011\end{array}$ & $\begin{array}{c}\tau \\
0.998 \\
0.017\end{array}$ & $\begin{array}{c}\alpha \\
0.003 \\
0.155\end{array}$ & $\begin{array}{c}\eta \\
0.240 \\
0.005\end{array}$ & $\begin{array}{c}\tau \\
0.999 \\
0.016\end{array}$ & $\begin{array}{c}\alpha \\
0.000 \\
0.033\end{array}$ & $\begin{array}{c}\eta \\
0.180 \\
0.007\end{array}$ & $\begin{array}{c}\tau \\
0.999 \\
0.010\end{array}$ & $\begin{array}{c}\alpha \\
0.005 \\
0.097\end{array}$ & $\begin{array}{c}\eta \\
0.240 \\
0.003\end{array}$ & $\begin{array}{c}\tau \\
1.000 \\
0.011\end{array}$ \\
\hline M & $\begin{array}{r}-0.005 \\
0.049\end{array}$ & $\begin{array}{l}0.180 \\
0.013\end{array}$ & $\begin{array}{l}0.995 \\
0.019\end{array}$ & $\begin{array}{l}0.001 \\
0.155\end{array}$ & $\begin{array}{l}0.239 \\
0.006\end{array}$ & $\begin{array}{l}0.996 \\
0.019\end{array}$ & $\begin{array}{l}0.002 \\
0.027\end{array}$ & $\begin{array}{l}0.179 \\
0.007\end{array}$ & $\begin{array}{l}0.999 \\
0.011\end{array}$ & $\begin{array}{l}0.004 \\
0.080\end{array}$ & $\begin{array}{l}0.240 \\
0.003\end{array}$ & $\begin{array}{l}0.999 \\
0.011\end{array}$ \\
\hline $\mathrm{C}$ & $\begin{array}{r}-0.005 \\
0.047\end{array}$ & $\begin{array}{l}0.179 \\
0.011\end{array}$ & $\begin{array}{l}0.998 \\
0.019\end{array}$ & $\begin{array}{l}0.004 \\
0.146\end{array}$ & $\begin{array}{l}0.240 \\
0.005\end{array}$ & $\begin{array}{l}0.999 \\
0.019\end{array}$ & $\begin{array}{l}0.001 \\
0.026\end{array}$ & $\begin{array}{l}0.179 \\
0.007\end{array}$ & $\begin{array}{l}1.000 \\
0.011\end{array}$ & $\begin{array}{l}0.002 \\
0.078\end{array}$ & $\begin{array}{l}0.240 \\
0.003\end{array}$ & $\begin{array}{l}1.000 \\
0.011\end{array}$ \\
\hline \multicolumn{13}{|c|}{ Gaussian, 8-nearest neighbor structure } \\
\hline $\mathrm{P}$ & -0.002 & 0.114 & 1.015 & -0.003 & 0.185 & 1.035 & -0.001 & 0.116 & 1.017 & 0.000 & 0.187 & 1.038 \\
\hline M & 0.000 & 0.113 & 1.013 & -0.004 & 0.186 & 1.031 & -0.001 & 0.116 & 1.016 & 0.001 & 0.188 & 1.037 \\
\hline $\mathrm{C}$ & 0.003 & 0.130 & 1.003 & -0.005 & 0.210 & 0.994 & -0.001 & 0.132 & 1.008 & 0.014 & 0.211 & 1.001 \\
\hline
\end{tabular}

SBEL.M and SBEL.C tests (with $b=1.5 n^{1 / 5}$ ) of the null (4-nearest neighbor conditional Gaussian) model under all the data generating-models in Table 1 . Table 3 displays the median parameter estimates from the SBEL methods in fitting the null model under the various data-generating models and, for comparison, includes estimates from standard maximum pseudo-likelihood applied to the null model.

These results indicate that all SBEL tests achieved proper sizes with both weaker and stronger levels of dependence. The greatest difference between nominal and observed values in Table 2 was $2.4 \%$ for SBEL.C at nominal level $10 \%$ with weak dependence on the smaller lattice $(30 \times 30)$. Of the 32 situations represented in Table 2, 6 had observed test sizes that differed from the nominal levels by more than $1 \%$, 3 that differed by more than $1.5 \%$, and only 1 that differed by more than $2 \%$. Maintenance of proper test size may also be seen in the top four panels of Figure 2 for which power is essentially equal to size for data generated from models having 4-nearest neighbor structure and either weak or strong dependence; recall that the fitted model for all situations depicted in Figure 2 assumed a 4-nearest neighbor structure.
The top four panels of Figure 2 show that SBEL.M was unable to detect departures from an assumed neighborhood structure of 4-nearest and an underlying true structure of 8nearest neighbors, while SBEL.C was able to detect this difference between true and assumed models. When lattice size was smaller $(30 \times 30)$ power was greater for SBEL.C when dependence was stronger (top left panel of Figure 2) but this difference was not evident for the larger lattice size (second panel in the first column of Figure 2). Fitted models are apparently able to reflect the behavior anticipated in higher moments even when fit to data using an incorrect neighborhood assumption. The summary of estimation presented in Table 3 indicates why this is the case. Estimated dependence parameters for fitted models using 4-nearest neighbors were larger than the actual values used in generating data from models with 8-nearest neighbors, which were $\eta=0.09$ for weak dependence and $\eta=0.12$ for strong dependence. Conditional expected values for the Gaussian models of this section involve the dependence parameter multiplied by the sum of deviations from marginal means at neighboring locations (see the beginning of Section 4). These deviations are similar among all neighbors, regardless of whether there 
Table 4. Actual sizes (expressed as percentages) of SBEL.M, SBEL.C and SBEL.C2 tests, at nominal levels of $10 \%, 5 \%$ and $1 \%$, for assessing the null (4-nearest neighbor conditional Gaussian) model when the data-generating model belongs to this model class with $\alpha=0, \tau=1$ and either weak $\eta=0.18$ or strong $\eta=0.24$ dependence parameters. The SBEL tests reported here used the block scaling $b=3 n^{1 / 5}$

\begin{tabular}{|c|c|c|c|c|c|c|c|c|c|c|c|c|}
\hline \multirow{2}{*}{ Nominal } & \multicolumn{6}{|c|}{$30 \times 30$ region } & \multicolumn{6}{|c|}{$50 \times 50$ region } \\
\hline & \multicolumn{3}{|c|}{ weak $\eta=0.18$} & \multicolumn{3}{|c|}{ strong $\eta=0.24$} & \multicolumn{3}{|c|}{ weak $\eta=0.18$} & \multicolumn{3}{|c|}{ strong $\eta=0.24$} \\
\hline$\overline{\text { SBEL.M }}$ & 7.2 & 4.1 & 1.7 & 7.3 & 4.0 & 1.4 & 6.6 & 2.8 & 0.4 & 6.1 & 2.8 & 0.4 \\
\hline SBEL.C2 & 8.2 & 4.0 & 2.0 & 8.0 & 4.5 & 2.0 & 6.6 & 2.5 & 0.3 & 6.2 & 2.3 & 0.3 \\
\hline
\end{tabular}

are 4 or 8 . Thus, fitting a model that assumes 4 -nearest neighbors to data generated using 8-nearest neighbors results in a smaller sum of neighboring deviations than the truth which, to reflect observed values, is then multiplied by a dependence parameter estimated as larger than the truth. This results, then, in estimated conditional expectations that are similar for both true and estimated models. The phenomenon evidenced in the first and second panels of the second column in Figure 2 is that this seems to also hold for higher conditional moments. In contrast, estimation cannot compensate for assumed model structures that incorrectly specify relations that should hold between sets of locations (i.e., conditional covariances in SBEL.C). The power of SBEL.C to detect an incorrect assumption of 4nearest neighbors when data are generated using 8-nearest neighbors is high, as evidenced in the first and second panels of the first column in Figure 2.

In marked contrast to the the ability of SBEL.C but not SBEL.M to detect incorrect assumptions of neighborhood structure, SBEL.M was better able to detect departures of distributional form than was SBEL.C, as shown in the lower four panels of Figure 2. Here, unless the assumed neighborhood structure also departed from the actual situation, SBEL.C was relatively ineffective in detecting an incorrectly specified distribution in the null model (third and fourth panels in the first column of Figure 2). The assessment of SBEL.M using higher moments, however, could always uncover this inadequacy in the assumed model.

In total, the values presented in Table 3 indicate that estimation using SBEL procedures is roughly equivalent to estimation using a single pseudo-likelihood function for an entire set of data. Median parameter estimates for both SBEL.C (labeled $\mathrm{C}$ in Table 3) and SBEL.M (labeled M in Table 3) are quite similar to estimates produced using pseudo-likelihood (values labeled $\mathrm{P}$ in Table 3). The similarity between SBEL and pseudo-likelihood in median absolute deviations between estimated and true parameter values when the assumed (fitted) and true models are the same also indicates that the incorporation of pseudo-likelihood estimating equations in an SBEL procedure produces essentially maximum pseudo-likelihood estimates. As would be anticipated, these median deviations were somewhat smaller for simulations conducted using the larger $50 \times 50$ lattice than for those conducted using the smaller $30 \times 30$ lattice.

Repeating the simulation results with a larger block scaling $b=3 n^{1 / 5}$ produced qualitatively similar results in both power curves and parameter estimates though the sizes of SBEL tests tended to be less accurate than with the scaling $b=1.5 n^{1 / 5}$. Table 4 shows the sizes of SBEL tests with $b=3 n^{1 / 5}$. In choosing the block scale $b$, there exists a tradeoff where blocks should be large enough to capture the underlying spatial dependence (among the evaluated spatial estimating functions), but overly large block scaling entails that fewer data blocks are available as input in defining the SBEL ratio (7). The latter degrades performance and, in our simulation studies, the block scaling $b=3 n^{1 / 5}$ reduced the number of available data blocks by roughly half compared to $b=1.5 n^{1 / 5}$. In this sense, a slightly worse performance with $b=3 n^{1 / 5}$ might be expected for the weakly dependent Markov models here. In practice, block scaling could potentially be chosen by computing SBEL ratio over a series of $b$ and then selecting from a range where the SBEL evaluations appear to stabilize, which is the "minimum volatility" method of block selection by Politis, Romano and Wolf [36] (sec. 9.3.2); see [29].

\section{ASSESSMENT OF CONDITIONAL BINARY MODELS}

In this section, we consider the SBEL method for model assessment in binary Markov models. The null model is taken to be conditional binary (i.e., auto-logistic) with a 4-nearest neighbor structure $\mathcal{N}(\mathbf{s})=\mathbf{s}+\mathcal{M}, \mathbf{s} \in \mathbb{Z}^{2}$ with $\mathcal{M}=\left\{(-1,0)^{\prime},(1,0)^{\prime},(0,1)^{\prime},(0,-1)^{\prime}\right\}$ as follows. In (2), the conditional distributional $X(\mathbf{s}) \mid\{X(\mathbf{t}): \mathbf{t} \in \mathcal{N}(\mathbf{s})\}$ is Bernoulli $p(\mathbf{s} ; \kappa, \eta)$ where

$$
\operatorname{logit}[p(\mathbf{s} ; \kappa, \eta)]=\operatorname{logit}(\kappa)+\eta \sum_{\mathbf{t} \in \mathcal{N}(\mathbf{s})}[X(\mathbf{t})-\kappa]
$$

and $\kappa \in(0,1)$ represents the Bernoulli mean in an independence model and $\eta \in \mathbb{R}$ is a dependence parameter; depending on the range of $\eta, \kappa$ will also essentially match the marginal mean of $X(\mathbf{s})$ (e.g. [6]). Here, the score-based estimating functions (3) are equivalent to 
$G_{p}\left[\mathcal{C}_{p}(\mathbf{s}) ; \kappa, \eta\right]=\left(\begin{array}{c}X(\mathbf{s})-p(\mathbf{s} ; \kappa, \eta) \\ {[X(\mathbf{s})-p(\mathbf{s} ; \kappa, \eta)] \sum_{\mathbf{t} \in \mathcal{N}(\mathbf{s})}[X(\mathbf{t})-\kappa]}\end{array}\right)$

where $p=2$ and $\mathcal{C}_{p}(\mathbf{s})=\{X(\mathbf{t}): \mathbf{t}=\mathbf{s}$ or $\mathbf{t} \in \mathcal{N}(\mathbf{s})\}$. For augmenting estimating functions, we will present results for two cases. One case involves

$$
\begin{aligned}
G_{a}\left[\mathcal{C}_{p}(\mathbf{s}) ; \kappa, \eta\right]= & \left([X(\mathbf{s})-p(\mathbf{s} ; \kappa, \eta)]^{2}\right. \\
& -p(\mathbf{s} ; \kappa, \eta)[1-p(\mathbf{s} ; \kappa, \eta)]) \sum_{\mathbf{t} \in \mathcal{N}(\mathbf{s})} X(\mathbf{t}),
\end{aligned}
$$

a (neighbor-sum-scaled) conditional variance, which results in a (conditional moment-based) test statistic referred to here as "SBEL.M." The other estimating function involves conditional covariances

$$
G_{a}\left[\mathcal{C}_{p}(\mathbf{s}), \mathcal{C}_{a}(\mathbf{s}) ; \kappa, \eta\right]=\sum_{\mathbf{t} \in \mathcal{N}^{a u g}(\mathbf{s})} X(\mathbf{t})[X(\mathbf{s})-p(\mathbf{s} ; \kappa, \eta)],
$$

with (similarly to the last section) a sum over all observations $\mathcal{C}_{a}(\mathbf{s})$ in an 8-nearest neighbor arrangement, not appearing in the 4-nearest neighbor scheme; we again refer to the resulting ("conditional covariance-based") SBEL test statistic as "SBEL.C." Both SBEL test statistics here have large sample $\chi_{1}^{2}$ distributions. We also tried formulations of test statistics with other estimating functions, such as using the conditional variance $[X(\mathbf{s})-p(\mathbf{s} ; \kappa, \eta)]^{2}-$ $p(\mathbf{s} ; \kappa, \eta)[1-p(\mathbf{s} ; \kappa, \eta)]$ alone or in combination with the conditional-variance-based $G_{a}\left[\mathcal{C}_{p}(\mathbf{s}) ; \kappa, \eta\right]$ above, or splitting the conditional covariance-based estimating function $G_{a}\left[\mathcal{C}_{p}(\mathbf{s}), \mathcal{C}_{a}(\mathbf{s}) ; \kappa, \eta\right]$ above into two sums (as in the last section). However, the results were qualitatively similar with

Table 5. Spatial data-generating models used in simulations to examine the performance of SBEL assessments of a fitted null conditional binary model. Dependence parameters listed correspond to $\eta$ in the conditional binary model or $\tilde{\eta}$ in the conditional Gaussian-based binary mixtures

\begin{tabular}{cccc}
\hline \hline Distribution & Neighbors & Weak & Strong \\
\hline Conditional Binary & 4-nearest & 0.5 & 0.8 \\
$\begin{array}{c}\text { Conditional Gaussian-based } \\
\text { Binary Mixture }\end{array}$ & 4-nearest & 0.18 & 0.24 \\
$\begin{array}{c}\text { Conditional Gaussian-based } \\
\text { Binary Mixture }\end{array}$ & 8-nearest & 0.09 & 0.12 \\
\hline
\end{tabular}

these variations and, to ease the presentation of results, we focus on the performance of SBEL.M and SBEL.C tests for the null model.

To evaluate the test statistics for the null model, we considered three spatial data-generating models based on conditional specifications, each with two parameter settings entailing weaker and stronger dependence. The first datagenerating model matched the null model structure with conditional Binary distributions, having a 4-nearest neighbor arrangement with $\kappa=0.5$ and varying the dependence parameter $\eta=0.5$ or 0.8 for weak or strong dependence. The two other models for data-generation involved a binary mixture distribution, described as follows. For each location $\mathbf{s} \in \mathcal{Z}^{2}$, a binary probability $\tilde{p}(\mathbf{s} ; \tilde{\eta})$ was generated as

$$
\operatorname{logit}[\tilde{p}(\mathbf{s} ; \tilde{\eta})]=\tilde{Z}(\mathbf{s}),
$$

where $\left\{\tilde{Z}(\mathbf{s}): \mathbf{s} \in \mathbb{Z}^{2}\right\}$ were values of a latent Markov random field process having conditional Gaussian distributions (as in the last section) with either a 4 - or 8-nearest neighborhood structure and parameters $(\tilde{\alpha}=0, \tilde{\tau}=1, \tilde{\eta})$, where the dependence parameter $\tilde{\eta}$ was varied to produce roughly weak or strong spatial dependence. Conditioned on the values $\tilde{p}(\mathbf{s} ; \tilde{\eta}) \in(0,1), \mathbf{s} \in \mathbb{Z}^{2}$, each observation $X(\mathbf{s})$ was then generated by an independent Bernoulli $\tilde{p}(\mathbf{s} ; \tilde{\eta})$ trial. Note that, because $\tilde{Z}(\cdot)$ has a marginal mean $\tilde{\alpha}=0$, the resulting $X(\cdot)$-observations have mean 0.5 , which roughly matches the marginal mean in the null data-generating model $(\kappa=0.5)$. That is, all data-generating models produce binary observations with essentially the same marginal mean of 0.5. These three data-generating models are summarized in Table 5.

For each model, we again generated data on two sampling regions, $30 \times 30$ and $50 \times 50$, using 2,000 simulations in every case. The SBEL test statistics were then computed using a block scaling of $b=1.5 n^{1 / 5}$ or $b=3 n^{1 / 5}$. With block scaling $b=1.5 n^{1 / 5}$, Table 6 presents the observed sizes of the SBEL tests, and Figure 3 displays power curves to compare SBEL.M and SBEL.C tests of the null (conditional binary 4-nearest neighbor) model for the three data generatingmodels in Table 5. Table 7 presents median parameter estimates from the SBEL methods in fitting the null model for each of the data-generating models, and again includes maximum pseudo-likelihood estimates for comparison.

The observed test sizes of Table 6 are not as close to nominal levels as were tests with the Gaussian conditionals

\begin{tabular}{|c|c|c|c|c|c|c|c|c|c|c|c|c|}
\hline \multirow[b]{3}{*}{ Nominal } & \multicolumn{6}{|c|}{$30 \times 30$ region } & \multicolumn{6}{|c|}{ 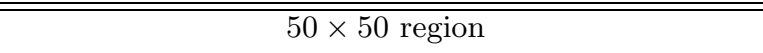 } \\
\hline & \multicolumn{3}{|c|}{ weak $\eta=0.5$} & \multicolumn{3}{|c|}{ strong $\eta=0.8$} & \multicolumn{3}{|c|}{ weak $\eta=0.5$} & \multicolumn{3}{|c|}{ strong $\eta=0.8$} \\
\hline & 10 & 5 & 1 & 10 & 5 & 1 & 10 & 5 & 1 & 10 & 5 & 1 \\
\hline$\overline{\text { SBEL.M }}$ & 7.7 & 3.4 & 0.7 & 9.6 & 4.6 & 0.4 & 8.5 & 4.2 & 0.7 & 9.5 & 4.6 & 1.1 \\
\hline SBEL.C & 11.5 & 6.1 & 1.3 & 11.4 & 5.6 & 1.1 & 11.6 & 5.7 & 1.1 & 11.5 & 5.9 & 0.9 \\
\hline
\end{tabular}

Table 6. Actual sizes (expressed as percentages) of SBEL.M and SBEL.C tests, at nominal levels of $10 \%, 5 \%$ and $1 \%$, for assessing the null conditional binary model when the data-generating model belongs to this model class with either weak $\eta=0.5$ or stronger $\eta=0.8$ dependence parameters. The SBEL tests reported here used block scaling $b=1.5 n^{1 / 5}$ 
Weak, $30 \times 30$, SBEL.C

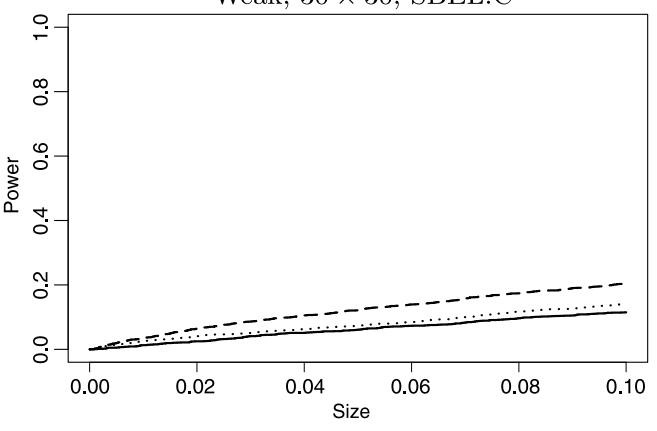

Strong, $30 \times 30$, SBEL.C

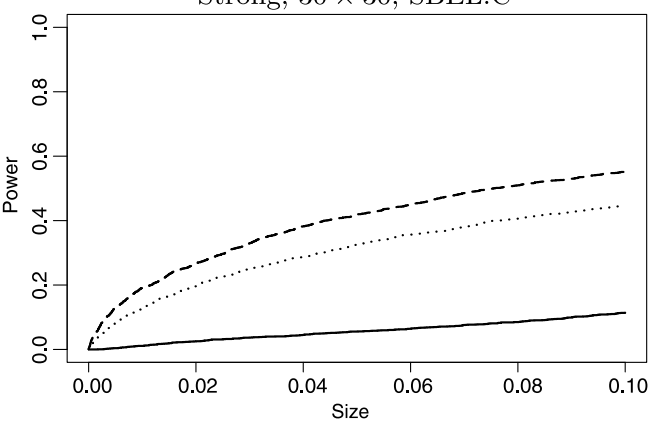

Weak, $50 \times 50$, SBEL.C

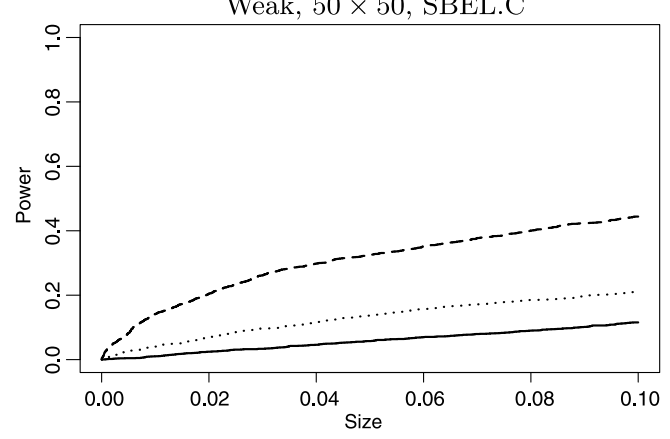

Strong, $50 \times 50$, SBEL.C

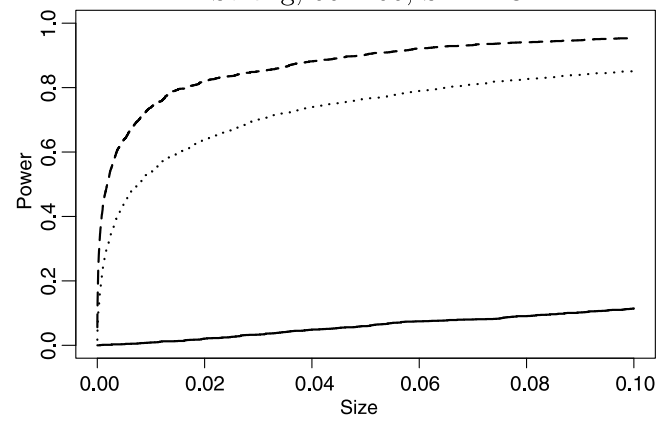

Weak, $30 \times 30$, SBEL.M

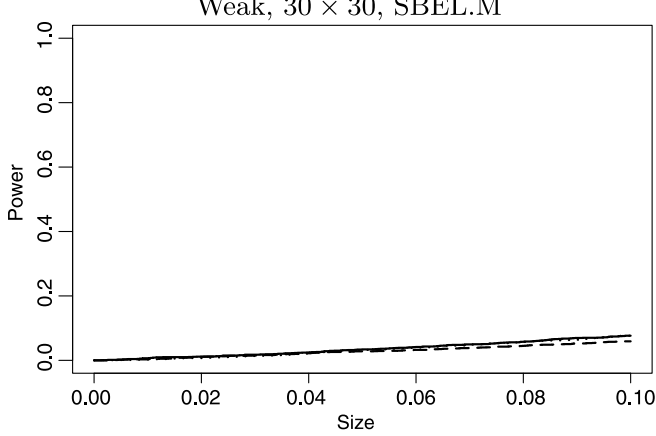

Strong, $30 \times 30$, SBEL.M

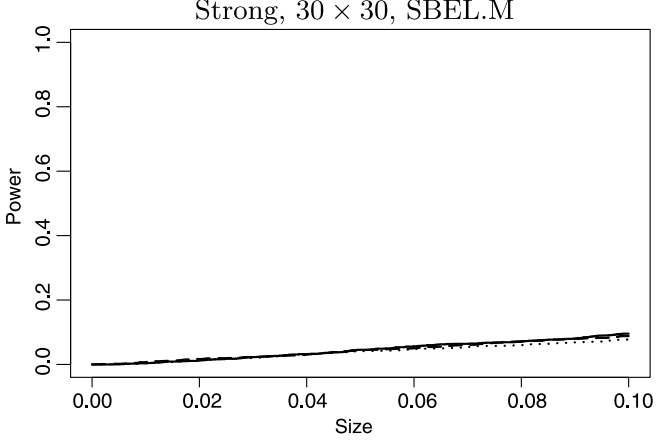

Weak, $50 \times 50$, SBEL.M

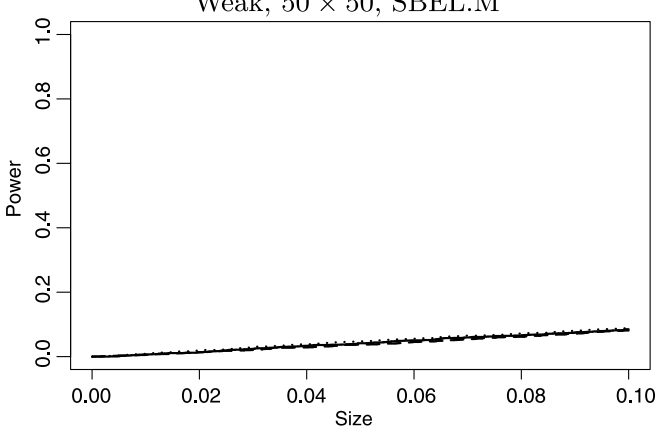

Strong, $50 \times 50$, SBEL.M

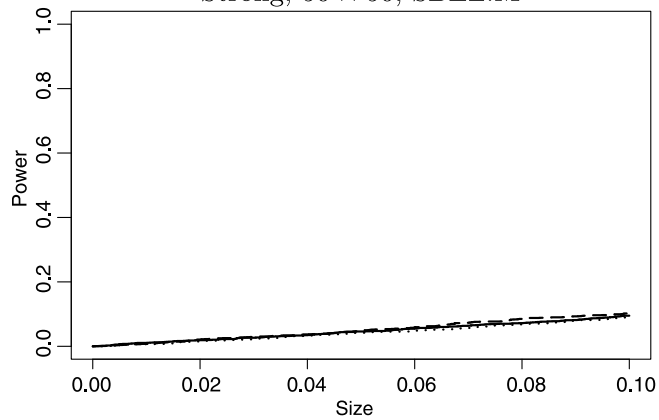

Figure 3. Power curves for testing the null (4-nearest neighbor conditional binary) model. Plots represent weak/strong dependence in data-generating models, SBEL tests $\left(b=1.5 n^{1 / 5}\right)$, and sampling regions; within a plot, curves correspond to data-generating models of 4-nearest neighbor conditional binary (-) or 4- and 8-nearest neighbor conditional Gaussian-based binary mixtures, denoted as $(\cdots)$ and $(--)$.

model, but are not terribly far out of line with those values either. Similarly to the Gaussian case, the observed test sizes did not seem to be influenced by the size of lattice or the strength of dependence. In contrast to this, power curves for testing the null model of conditional binary distributions with a 4-nearest neighbor structure were impacted by both the size of lattice and the strength of dependence used in data generation. The graphs of Figure 3 indicate that, for the data generating models investigated, SBEL.M proved ineffectual in distinguishing between the conditional binary 
Table 7. Median parameter estimates for $(\kappa, \eta)$ in fitting the null (4-nearest neighbor conditional binary) model with regular maximum pseudo-likelihood $(P)$ and maximum SBEL estimates from SBEL.M (M) and SBEL.C (C) functions (7) with $b=1.5 n^{1 / 5}$; these are divided by different data-generating models involving weak and strong dependence and different sampling region sizes. In the case where data-generation occurs under the null model, median absolute deviations from the true parameters ( $\kappa=0.5, \eta=0.5$ (weak) or 0.8 (strong)) appear in italics

\begin{tabular}{|c|c|c|c|c|c|c|c|c|}
\hline \multicolumn{9}{|c|}{ Conditional binary, 4-nearest neighbor } \\
\hline & \multicolumn{4}{|c|}{$30 \times 30$ region } & \multicolumn{4}{|c|}{$50 \times 50$ region } \\
\hline & \multicolumn{2}{|c|}{ weak dependence } & \multicolumn{2}{|c|}{ strong dependence } & \multicolumn{2}{|c|}{ weak dependence } & \multicolumn{2}{|c|}{ strong dependence } \\
\hline \multirow{3}{*}{$\mathrm{P}$} & $\kappa$ & $\eta$ & $\kappa$ & $\eta$ & $\kappa$ & $\eta$ & $\kappa$ & $\eta$ \\
\hline & 0.499 & 0.495 & 0.501 & 0.799 & 0.499 & 0.499 & 0.499 & 0.798 \\
\hline & 0.017 & 0.064 & 0.037 & 0.064 & 0.011 & 0.038 & 0.022 & 0.038 \\
\hline \multirow[t]{2}{*}{$\mathrm{M}$} & 0.501 & 0.495 & 0.499 & 0.798 & 0.501 & 0.500 & 0.4985 & 0.796 \\
\hline & 0.018 & 0.070 & 0.0420 & 0.070 & 0.011 & 0.042 & 0.023 & 0.042 \\
\hline \multirow[t]{3}{*}{$\mathrm{C}$} & 0.500 & 0.491 & 0.500 & 0.793 & 0.500 & 0.499 & 0.499 & 0.796 \\
\hline & 0.012 & 0.072 & 0.040 & 0.066 & 0.011 & 0.039 & 0.022 & 0.039 \\
\hline & \multicolumn{8}{|c|}{ Conditional Gaussian-based binary mixture, 4-nearest neighbor } \\
\hline $\mathrm{P}$ & 0.500 & 0.157 & 0.500 & 0.344 & 0.500 & 0.164 & 0.500 & 0.354 \\
\hline M & 0.501 & 0.153 & 0.498 & 0.350 & 0.500 & 0.165 & 0.500 & 0.354 \\
\hline $\mathrm{C}$ & 0.500 & 0.160 & 0.500 & 0.366 & 0.500 & 0.169 & 0.500 & 0.377 \\
\hline \multicolumn{9}{|c|}{ Conditional Gaussian-based binary mixture, 8-nearest neighbor } \\
\hline $\mathrm{P}$ & 0.500 & 0.104 & 0.501 & 0.242 & 0.500 & 0.108 & 0.500 & 0.252 \\
\hline M & 0.500 & 0.103 & 0.501 & 0.242 & 0.501 & 0.106 & 0.500 & 0.255 \\
\hline $\mathrm{C}$ & 0.500 & 0.104 & 0.501 & 0.257 & 0.501 & 0.112 & 0.500 & 0.266 \\
\hline
\end{tabular}

model and its mixture counterparts with either 4-nearest or 8-nearest neighbors, while SBEL.C was able to detect departures from the null conditional binary with 4-nearest neighbors. The power of SBEL.C was low $(\leq 0.2)$ when data were generated on the smaller lattice $(30 \times 30)$ with weaker dependence $(\eta=0.50)$. Increasing dependence $(\eta=0.80)$ more than doubled power across the entire range of sizes (compare the first and second panels in the left column of Figure 3). Increasing lattice size to $50 \times 50$ had a similar, but perhaps less dramatic, effect (compare the first and third panels in the left column of Figure 3). To achieve power greater than 0.75 for some test sizes less than 0.10 required both a larger $50 \times 50$ lattice and stronger dependence of $\eta=0.80$ (see the fourth panel in the left column of Figure 3).

The estimation results reported in the upper portion of Table 7 again indicate that using pseudo-likelihood estimating equations in SBEL procedures produces estimates that are similar to straight-up maximum pseudo-likelihood using data from the entire lattice to construct only one objective function. Fitting the null model of conditional binary distributions having 4-nearest neighbors to data generated from Gaussian-binary mixtures resulted in estimates that correctly reflect the marginal binary probability $(0.50)$ that is the expected value in both the Gaussian Markov random field and the resulting spatial mixture. It is not clear what the dependence parameter of the null model should be for these mixture data sets, and we do not attach any particular meaning to correspondence or lack of correspondence between values of $\eta$ in the conditional Gaussian mixing distributions used to generate data and the estimated values of $\eta$ for the conditional binary model used for estimation. Parameter estimates of $\eta$ for the null model, however, do correctly reflect the the weaker and stronger dependencies used in data generating models.

Again, repeating the simulation results with a larger block size $b=3 n^{1 / 5}$ produced qualitatively similar, although slightly less accurate, results to the block choice of $b=1.5 n^{1 / 5}$. We illustrate this in Table 8 by showing the median estimates and estimation errors under the null datagenerating model, which compare favorably with the values of Table 7 .

\section{VERIFYING NEIGHBORHOOD SELECTION IN MODELING PLANT DISEASE}

As an example of the use of the SBEL methods proposed here we consider the selection of neighborhoods in a binary Markov random field model for presence/absence of a plant disease in an agricultural field. Bean pod mottle virus (BPMV) is a disease that affects soybeans and other legumes. It can decrease both the yield and the quality of soybeans and there are no known varieties of soybeans that are completely resistant to BPMV [13]. Transmission of BPMV is believed to occur through an insect vector of leaf eating beetles and the pattern, if any, of how the virus is spatially distributed in fields is of interest to plant pathologists and agronomists in attempting to develop controls for spread of the disease. Figure 4 shows one 
Table 8. Median parameter estimates for $(\kappa, \eta)$ in fitting the null (4-nearest neighbor conditional binary) model with maximum SBEL estimates from SBEL.M (M) and SBEL.C (C) functions (7) with $b=3 n^{1 / 5}$, when the null model is true for data-generation. Median absolute deviations from the true parameters $(\kappa=0.5, \eta=0.5$ (weak) or 0.8 (strong)) appear in italics

\begin{tabular}{|c|c|c|c|c|c|c|c|c|}
\hline & & & & binary & st neigl & & & \\
\hline & & & gion & & & & gion & \\
\hline & we & dence & stro & dence & wea & dence & stro & dence \\
\hline & $\kappa$ & $\eta$ & $\kappa$ & $\eta$ & $\kappa$ & $\eta$ & $\kappa$ & $\eta$ \\
\hline M & 0.500 & 0.498 & 0.499 & 0.806 & 0.500 & 0.501 & 0.499 & 0.798 \\
\hline & 0.021 & 0.0802 & 0.047 & 0.077 & 0.013 & 0.048 & 0.025 & 0.048 \\
\hline $\mathrm{C}$ & 0.499 & 0.489 & 0.499 & 0.793 & 0.500 & 0.499 & 0.498 & 0.796 \\
\hline & 0.022 & 0.081 & 0.045 & 0.078 & 0.013 & 0.044 & 0.025 & 0.044 \\
\hline
\end{tabular}

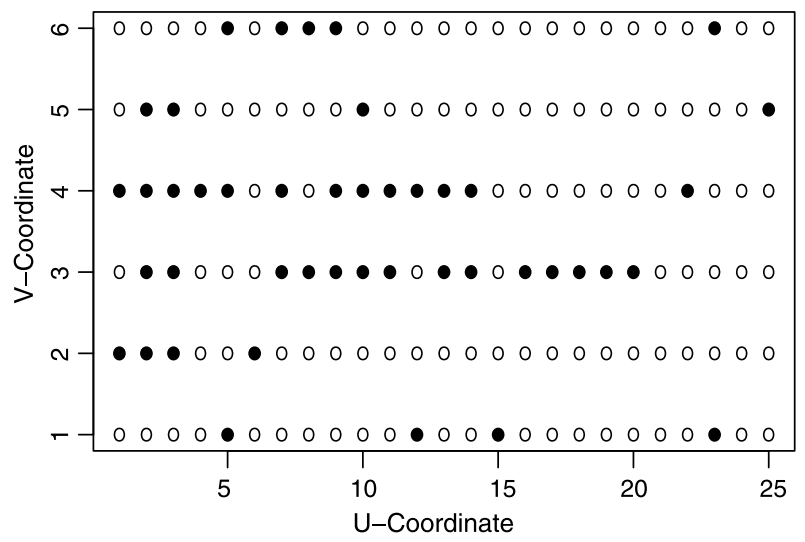

Figure 4. Presence (solid symbol) and absence (open symbol) of bean pod mottle virus in a soybean field.

soybean field, from a study at Iowa State University, divided into 150 quadrats as a lattice having 6 rows and 25 columns. While the scaling of Figure 4 is not proportional to the true physical situation, it is true that locations were somewhat closer together in the horizontal direction (which corresponded to within agricultural rows) than in the vertical direction (which corresponded to across agricultural rows). Each quadrat contained four plants, and the disease was recorded as present or absent in each quadrat. A binary Markov random field model was formulated for analysis of these data in the following manner. For a spatial location $\mathbf{s}=(u, v)^{\prime}$, where $u$ is an integer-valued horizontal coordinate and $v$ an integer-valued vertical coordinate, define a random variable $Y(\mathbf{s})=1$ if BPMV was present at the location and $Y(\mathbf{s})=0$ otherwise. Given its neighborhood $\mathcal{N}(\mathbf{s})$, each location is assigned a conditional binary probability mass function $f_{\theta}(Y(\mathbf{s}) \mid\{Y(\mathbf{t}): \mathbf{t} \in \mathcal{N}(\mathbf{s}))$ under the Markov assumption described earlier as, with parameters $\theta=(\kappa, \eta)^{\prime} \in(0,1) \times \mathbb{R}$ and

$$
\begin{aligned}
\operatorname{logit} & {\left[f_{\theta}(Y(\mathbf{s})=1 \mid\{Y(\mathbf{t}): \mathbf{t} \in \mathcal{N}(\mathbf{s}))]\right.} \\
& =\log \left(\frac{\kappa}{1-\kappa}\right)+\eta \sum_{\mathbf{t} \in \mathcal{N}(\mathbf{s})}\{Y(\mathbf{t})-\kappa\} .
\end{aligned}
$$

Based on the visual impression of Figure 4 and knowledge that spacing was somewhat wider among than within rows, neighborhoods were specified as $\mathcal{N}(\mathbf{s})=\left\{\mathbf{s}+(u, v)^{\prime}: u=\right.$ $\pm 1, v=0\}$, that is, adjacent locations but within rows only. This definition of neighborhoods is arbitrary, and we would like to have some justification for the choice made. A natural alternative would be a four-nearest neighbor structure, which is commonly used in agricultural applications. It is this alternative that we wish to assess.

Because of the neighborhood structure specified, which can be described as a two-nearest within-row structure, for initial fitting purposes, we defined $G_{p}\left[\mathcal{C}_{p}(\mathbf{s}) ; \theta\right]$ as in (3) with $p=2$ using a border strip consisting of the rightmost and left-most data columns in Figure 4. That is, the sampling region $D_{n}$ of Section 3 was all locations shown in Figure 4, while the sampling sites $\mathcal{S}_{n}$ for $\mathcal{C}_{p}(\mathbf{s})$ were all locations except for the two end columns (c.f., Figure 1). The resulting SBEL estimates were $\hat{\kappa}=0.257$ with a $90 \%$ interval of $(0.107,0.736)$, and $\hat{\eta}=1.549$ with a $90 \%$ interval of $(0.843,2.357)$ using a block size $b=3 \approx n^{1 / 5}$ with $n=150$. The block size here follows the rule of thumb given by Nordman [29] and the simulations of Sections 4 and 5 . To test our selected neighborhood specification against an alternative of four-nearest neighbors, the augmentation neighborhood of Section 3 was taken to be the two-nearest north and south locations $\mathcal{N}^{\text {aug }}(\mathbf{s})=$ $\left\{\mathbf{s}+(u, v)^{\prime}: u=0, v= \pm 1\right\}$ which constitute observations in a four-nearest neighborhood structure not already appearing in $\mathcal{N}(\mathbf{s})$. Using SBEL.C with this augmented neighborhood gave a procedure analogous to the comparison of four-nearest and eight-nearest neighbors in the previous simulations, with one minor difference for simplicity. Namely, we defined the additional covariance-type estimating function as $G_{a}[\mathcal{C}(\mathbf{s}) ; \theta]=\left[Y(\mathbf{s})-\mu_{1}(\mathbf{s}, \theta)\right] M(\mathbf{s}), \mathbf{s} \in \mathcal{S}_{n}$, using the sample mean $M(\mathbf{s})=\sum_{\mathbf{t} \in \mathcal{N}^{a u g}(\mathbf{s}) \cap \mathcal{S}_{n}} X(\mathbf{t}) /\left|\mathcal{N}^{\text {aug }}(\mathbf{s}) \cap \mathcal{S}_{n}\right|$ of augmented neighboring observations of location $\mathbf{s}$ within $\mathcal{S}_{n}$, which is similar to (6) and has mean zero under the specified neighborhood structure, but does not create additional border strips from the first and last rows of Figure 4 . The test of a two-nearest within-row neighborhood structure against the alternative of four-nearest neighbors 
resulted in a $\mathrm{p}$-value of 0.768 , providing justification for the selection of within-row neighbors. In this application, the pattern of BPMV within the field is highly structured in a manner that can be represented as statistical dependence within rows.

\section{DISCUSSION}

Applying Markov random field models to problems that involve spatial structure requires that a number of choices be made in the process of model formulation. One must select distributional forms for conditional density or mass functions used to represent responses, the neighborhood structure that dictates conditional dependencies, whether or not to include a large-scale model component such as trend, and the manner in which dependence is assumed to have influence (e.g., unidirectional, directional, or time-varying). The number of tools available to help guide these decisions is increasing, such as the model-based exploratory diagnostic called the S-value by Kaiser and Caragea [17]. Similarly, overall assessment of the plausibility that a given model could have led to data of the type observed in a problem is more feasible than in the past through the use of tools such as the generalized spatial residuals and accompanying tests of Kaiser, Lahiri and Nordman [20], which may be considered ubiquitous goodness of fit procedures. In this article we have provided development of practical procedures for assessing model structures that are more closely connected with particular modeling choices that must be made. These procedures are based on the theory of spatial blockwise empirical likelihood (SBEL in this article) developed by Nordman [29], and their efficacy in model assessment hinges on the selection of estimating functions in the form of moment conditions to reflect particular aspects of assumed model behavior. For example, conditional covariances computed for sets of locations that, under an assumed model, should be conditionally independent (called SBEL.C in this article) are effective in detecting departures from the neighborhood structure used in model construction. The intelligent construction of augmentation neighborhoods and associated estimating functions to arrive at the set of equations $G_{a}\left[C_{p}(\mathbf{s}), C_{a}(\mathbf{s})\right]$ of Section 2.2 has potential for detection of departures from a variety of particular modeling assumptions such as the small-scale structure assumed in a model (e.g., unidirectional dependence) or an assumption of constant conditional variance (a common assumption for models with Gaussian conditionals). The use of conditional moments and conditional covariances with non-neighbors used in this article only scratches the surface of what may be possible.

Because they depend critically on the selection of testing conditions, SBEL procedures function in an arena that lies somewhere between model selection and goodness of fit tests. They provide a test procedure for specific questions about assumed model structures and in this sense provide goodness of fit results. At the same time, they provide such results only about the particular aspects of model behavior embodied in the augmentation estimating functions (or testing conditions) selected for use and are thus not necessarily indicative of overall model performance. Ubiquitous goodness of fit tests provide useful indications of whether or not a fitted model could be viewed as a reasonable "data generating mechanism" for a set of observed values. And statistical estimation can be viewed as a process that, under a chosen criterion, optimizes the ability of the model structure to reflect data values. Overall goodness of fit procedures, then, can only provide a global picture of the adequacy of a model. While this is important, some problems demand a more detailed assessment of model structure, given a prerequisite of overall plausibility. A simple example is provided by the conditional Gaussian models of Section 4. If data are simulated from such a model with an underlying 8-nearest neighborhood structure but a model assuming 4-nearest neighbors is fit to those data, the fitted model will provide estimates of conditional expectations that are nearly the same as would be obtained by fitting a model with 8-nearest neighbors. Given correct assumptions of constant conditional variance and normal distributions, then, the overall goodness of fit procedures of [20] will not reject a 4-nearest neighborhood structure as a plausible model. The model fitted using an incorrect neighborhood assumption represents conditional distributions at each location quite well. For this same reason, SBEL procedures based on conditional moments (SBEL.M in this article) will not provide any indication of model inadequacy. But, an SBEL procedure based on conditional covariances between locations that are not (assumed) neighbors of each other will detect the incorrect assumption of neighborhood structure. The question, then, is whether the model fitted with 4-nearest neighbors is "adequate" or "inadequate," and the answer clearly depends on what use is being made of that model. If the concern is estimation of conditional distributions at each location, then the fitted model must be considered adequate. But if the concern is the extent of spatial structure that can be represented through a lack of conditional independence, then the fitted model must be considered inadequate. The SBEL methodology presented in this article provides a formal approach to answering questions of adequacy for specific aspects of model behavior that may be important in a given application. As such, SBEL procedures compliment both modelbased diagnostics that may motivate choices in model formulation, and overall goodness of fit procedures that assess fitted models from a global viewpoint.

\section{ACKNOWLEDGEMENTS}

The authors are grateful to an associate editor and reviewer for helpful comments that improved the manuscript. Data for the bean pod mottle virus example was provided by 
Forrest Nutter, Department of Plant Pathology, Iowa State University.

\section{Received 3 January 2012}

\section{REFERENCES}

[1] Arnold, B. C., Castillo, E. and Sarabia, J. M. (1992). Conditionally specified distributions. Lect. Notes Statist., 73. Springer. MR1177398

[2] BesAG, J. (1974). Spatial interaction and the statistical analysis of lattice systems (with discussion). J. R. Stat. Soc. B 36192 236. MR0373208

[3] Besag, J. (1975). Statistical analysis of non-lattice data. The Statistician 24 179-195.

[4] Bravo, F. (2005). Blockwise empirical entropy tests for time series regressions. J. Time Ser. Anal. 26 185-210. MR2122895

[5] Bravo, F. (2009). Blockwise generalized empirical likelihood inference for non-linear dynamic moment conditions models. Econometrics Journal 12 208-231. MR2562384

[6] Caragea, P. C. and Kaiser, M. S. (2009). Autologistic models with interpretable parameters. J. Agric. Biol. Environ. Stat. 14 281-300. MR2750841

[7] Carlstein, E. (1986). The use of subseries methods for estimating the variance of a general statistic from a stationary time series. Ann. Statist. 14 1171-1179. MR0856813

[8] Chunang, C. and Chan, N. H. (2002). Empirical likelihood for autoregressive models, with applications to unstable time series. Statist. Sinica 12 387-407. MR1902716

[9] Chen, S. X. and Wong, C. (2009). Smoothed block empirical likelihood for quantiles of weakly dependent processes. Statist. Sinica 19 71-82. MR2487878

[10] Chen, S. X., Härdle, W. and Li, M. (2003). An empirical likelihood goodness-of-fit test for time series. J. R. Stat. Soc. B 65 663-678. MR1998627

[11] Cressie, N. (1993). Statistics for Spatial Data, 2nd ed. John Wiley \& Sons, New York. MR1239641

[12] Doukhan, P. (1994). Mixing: Properties and examples. Lecture Notes in Statistics, 85. Springer, New York. MR1312160

[13] Gieser, L. J., Ghabrial, S. A., Hunt, T. E. and Hill, J. H. (2002). Bean pod mottle virus: a threat to U.S. soybean production. Plant Disease 86 1280-1289.

[14] Guan, Y., Sherman, M. and Calvin, J. A. (2004). A nonparametric test for spatial isotropy using subsampling. J. Amer. Statist. Assoc. 99 810-821. MR2090914

[15] Guyon, X. (1995). Random Fields on a Network. SpringerVerlag, New York. MR1344683

[16] Hardouin, C. and YAO, J. F. (2008). Multi-parameter automodels with applications to cooperative systems and analysis of mixed state data. Biometrika 95 335-349. MR2521588

[17] Kaiser, M. S. and Caragea, P. C. (2009). Exploring dependence with data on spatial lattices. Biometrics 65 857-865 MR2649858

[18] Kaiser, M. S. and Cressie, N. (1997). Modeling Poisson variables with positive spatial dependence. Statist. Probab. Lett. 35 423-432. MR1483030

[19] Kaiser, M. S., Cressie, N., and Lee, J. (2002). Spatial mixture models based on exponential family conditional distributions. Statist. Sinica 12 449-474. MR1902719

[20] Kaiser, M. S., Lahiri, S. N. and Nordman, D. J. (2012). Goodness of fit tests for a class of Markov random field models. Ann. Statist. 40 104-130.

[21] Kitamura, Y. (1997). Empirical likelihood methods with weakly dependent processes. Ann. Statist. 25 2084-2102. MR1474084
[22] Kitamura, Y., Tripathi, G. and Ahn, H. (2004). Empirical likelihood-based inference in conditional moment restriction models. Econometrica 72 1667-1714. MR2095529

[23] KüNsch, H. R. (1989). The jackknife and the bootstrap for general stationary observations. Ann. Statist. 17 1217-1241. MR1015147

[24] LahiRi, S. N. (2003). Resampling Methods for Dependent Data. Springer, New York. MR2001447

[25] LEI, Q. and QIN, Y. (2011). Empirical likelihood for quantiles under negatively associated samples. J. Statist. Plann. Inference 141 1325-1332. MR2739173

[26] Liu, R. Y. and Singh, K. (1992). Moving blocks jackknife and bootstrap capture weak dependence. In Exploring the Limits of the Bootstrap (Edited by R. Lepage and L. Billard) 225-248. Wiley, New York. MR1197787

[27] Li, B., Genton, M. G. and Sherman, M. (2008). On the asymptotic joint distribution of sample space-time covariance estimators. Bernoulli 14 228-248. MR2401661

[28] Newey, W. K. and Smith, R. J. (2004). Higher order properties of GMM and generalized empirical likelihood estimators. Econometrica 72 219-255. MR2031017

[29] Nordman, D. J. (2008). A blockwise empirical likelihood for spatial data. Statist. Sinica 18 1111-1129. MR2440078

[30] Nordman, D. J. and Caragea, P. C. (2008). Point and interval estimation of variogram models using spatial empirical likelihood. J. Amer. Statist. Assoc. 103 350-361. MR2420238

[31] Nordman, D. J., Sibbertsen, P. and Lahiri, S. N. (2007) Empirical likelihood for the mean under long-range dependence. J. Time Ser. Anal. 28 576-599. MR2396631

[32] Owen, A. B. (1988). Empirical likelihood ratio confidence intervals for a single functional. Biometrika 75 237-249. MR0946049

[33] Owen, A. B. (1990). Empirical likelihood confidence regions. Ann. Statist. 18 90-120. MR1041387

[34] Owen, A. B. (2001). Empirical Likelihood. Chapman \& Hall, London.

[35] Politis, D. N. and Romano, J. P. (1994). Large sample confidence regions based on subsamples under minimal assumptions. Ann. Statist. 22 2031-2050. MR1329181

[36] Politis, D. N., Romano, J. P., and Wolf, M. (1999). Subsampling. Springer, New York. MR1707286

[37] Qin, J. and Lawless, J. (1994). Empirical likelihood and general estimating equations. Ann. Statist. 22 300-325. MR1272085

[38] Qin, J. and LAwless, J. (1995). Estimating equations, empirical likelihood and constraints on parameters. Canad. J. Statist. 23 145-159. MR1345462

[39] QIN, Y. and Li, Y. (2011). Empirical likelihood for linear models under negatively associated errors. J. Multivariate Anal. 102 153163. MR2729427

[40] Qin, Y., Li, Y., YAnG, W. and LeI, Q. (2011). Confidence intervals for nonparametric regression functions under negatively associated errors. J. Nonparametr. Stat. 23 645-659. MR2836282

[41] Rue, H. and Held, L. (2005). Gaussian Markov Random Fields: Theory and Applications. Boca Raton: Chapman and Hall/CRC Press. MR2130347

[42] Sherman, M. (1996). Variance estimation for statistics computed from spatial lattice data. J. R. Stat. Soc. B 58 509-523. MR1394363

[43] WU, R. and CAO, J. (2011). Blockwise empirical likelihood for time series of counts. J. Multivariate Anal. 102 661-673. MR2755022

[44] Zhang, J. (2006). Empirical likelihood for NA series. Statist Probab. Lett. 76 153-160. MR2233388

[45] Zhu, J. and Morgan, G. D. (2004). Comparison of spatial variables over subregions using a block bootstrap. J. Agric. Biol. Environ. Stat. 9 91-104. 
Mark S. Kaiser

Department of Statistics

lowa State University

Ames, IA 50010

USA

E-mail address: mskaiser@iastate.edu
Daniel J. Nordman

Department of Statistics

lowa State University

Ames, IA 50010

USA

E-mail address: dnordman@iastate.edu 